\title{
The Enduring Scandal of Deduction
}

\section{Is Propositional Logic Really Uninformative?}

\author{
Marcello D'Agostino \\ Dipartimento di Scienze Umane Università di Ferrara (dgm@unife.it) \\ Luciano Floridi \\ Department of Philosophy, University of Hertfordshire and Faculty of Philosophy \\ and OUCL, University of Oxford (1.floridi@herts.ac.uk)
}

\begin{abstract}
Deductive inference is usually regarded as being "tautological" or "analytical": the information conveyed by the conclusion is contained in the information conveyed by the premises. This idea, however, clashes with the undecidability of firstorder logic and with the (likely) intractability of Boolean logic. In this article, we address the problem both from the semantic and the proof-theoretical point of view and propose a hierarchy of propositional logics that are all tractable (i.e. decidable in polynomial time), although by means of growing computational resources, and converge towards classical propositional logic. The underlying claim is that this hierarchy can be used to represent increasing levels of "depth" or "informativeness" of Boolean reasoning. Special attention is paid to the most basic logic in this hierarchy, the pure "intelim logic", which satisfies all the requirements of a natural deduction system (allowing both introduction and elimination rules for each logical operator) while admitting of a feasible (quadratic) decision procedure. We argue that this logic is "analytic" in a particularly strict sense, in that it rules out any use of "virtual information", which is chiefly responsible for the combinatorial explosion of standard classical systems. As a result, analyticity and tractability are reconciled and growing degrees of computational complexity are associated with the depth at which the use of virtual information is allowed.
\end{abstract}

Keywords: Boolean Logic, Tractability, Semantic Information, Analytical Reasoning, Natural Deduction

\section{Introduction: the enduring scandal of deduction}

Traditionally, there are two ways to explain the function of deductive inference:

1. Deductive inference governs truth-transmission. The set of all possible worlds that make (the conjunction of) the premises true is included in the set of possible worlds that make the conclusion true.

2. Deductive inference governs the "information flow". The information conveyed by the conclusion is contained in the information conveyed by (the conjunction of) the premises.

(C) 2008 Kluwer Academic Publishers. Printed in the Netherlands.

DagostinoFloridi-kl.tex; 8/09/2008; 15:00; p.1 
In classical logic, (1) and (2) are reconciled by means of a somewhat controversial notion of "semantic information", which makes the information conveyed by a sentence equal to the set of all the possible worlds ruled out by that sentence. The basic idea is simple and can be briefly explained as follows.

Intuitively, information goes hand in hand with unpredictability. More precisely, the Inverse Relationship Principle (IRP), as Barwise labelled it, states that there is an inverse relation between the probability of $P$ - which may range over sentences of a given language (as in (Carnap and Bar-Hillel, 1953) and (Bar-Hillel, 1964)) or events, situations or possible worlds (as in (Dretske, 1981)) — and the amount of semantic information carried by $P$. More formally, the semantic content (CONT) of $P$ is measured as the complement of the a priori probability of $P$ :

$$
\operatorname{CONT}(P)=1-\operatorname{Pr}(P)
$$

CONT does not satisfy the two requirements of additivity and conditionalization, which are satisfied by another measure, the informativeness (INF) of $P$, which is calculated as the reciprocal of $\operatorname{Pr}(P)$, expressed in bits:

$$
\operatorname{INF}(P)=\log \frac{1}{1-\operatorname{CONT}(P)}=-\log \operatorname{Pr}(P)
$$

Nowadays, one often translates IRP modally, by stating that the semantic information conveyed by $P$ is the set of all possible worlds, or (more cautiously) the set of all the descriptions of the relevant possible states of the universe, that are excluded by, or are inconsistent with, $P$. IRP has been assumed as uncontroversial at least since Popper, who is often credited as the first philosopher to have advocated it explicitly ((Popper, 1934)). And it has provided the basis for systematic attempts to develop a formal analysis of semantic information since Shannon's breakthrough ((Shannon and Weaver, 1949)).

Unfortunately, any analysis of semantic information that subscribes to IRP runs into two main difficulties: the Bar-Hillel-Carnap Paradox ((Floridi, 2004)) and the "scandal of deduction" ((Hintikka, 1973)). In this paper, we shall be concerned exclusively with the latter, but a brief outline of the former will help to set the discussion into the right context.

Following IRP, the less probable or possible $P$ is, the more semantic information $P$ is assumed to be carrying. It follows that contradictions - which describe impossible states, or states whose probability is 0 are the sort of messages that contain the highest amount of semantic information. This is conceptually unpalatable, to say the least. BarHillel and Carnap (Carnap and Bar-Hillel, 1953) were among the first 
to make explicit this prima facie counterintuitive inequality but also to bite the bullet:

It might perhaps, at first, seem strange that a self-contradictory sentence, hence one which no ideal receiver would accept, is regarded as carrying with it the most inclusive information. It should, however, be emphasized that semantic information is here not meant as implying truth. A false sentence which happens to say much is thereby highly informative in our sense. Whether the information it carries is true or false, scientifically valuable or not, and so forth, does not concern us. A self-contradictory sentence asserts too much; it is too informative to be true. (p. 229).

Since its formulation, the Bar-Hillel-Carnap paradox has been recognised as an unfortunate, yet perfectly correct and logically inevitable consequence of any quantitative theory of weakly semantic information (TWSI; "weakly" because truth-values play no role in it, as the passage cited above makes explicit). As a consequence, the problem has often been either ignored or tolerated as the price of an otherwise valuable approach. Recently, a solution to the paradox has been proposed ${ }^{1}$ which consists in showing that TWSI is based on a semantic principle that is too weak, namely the assumption that truth-values supervene on semantic information. A semantically stronger approach, according to which information encapsulates truth, can avoid the paradox and is more in line with the ordinary conception of what generally counts as factual information. However, even assuming a strongly semantic approach, the traditional theory of semantic information is still challenged by another highly counterintuitive consequence.

Consider what happens when we follow IRP in the opposite direction. Make a statement more and more likely and you gradually decrease its informational content. In the end, when the statement has probability 1 , or is true in all possible worlds, it conveys no information at all, and so all logical truths, being tautologies, become equally uninformative. But in classical logic, a sentence $Q$ is deducible from a finite set of premises $P_{1}, \ldots, P_{n}$ if and only if the conditional $P_{1} \wedge \ldots \wedge P_{n} \rightarrow Q$ is a tautology. Accordingly, since tautologies carry no information at all, no logical inference can yield an increase of information. Indeed, by identifying the semantic information carried by a sentence with the set of all possible worlds it excludes, it can be immediately recognized that, in any valid deduction, the information carried by the conclusion is contained in the information carried by the (conjunction of) the

\footnotetext{
${ }^{1}$ ((Floridi, 2004) and (Floridi, 2005), see (Bremer and Cohnitz, 2004) chap. 2 for an overview)
} 
premises. This is what is often meant by saying that tautologies and inferences are "analytical". 2

As pointed out by Dummett, the approach just described stems from an ultra-justificationist attitude, which seeks to obtain a bullet-proof notion of deduction that leaves no room for sceptical attacks:

Once the justification of deductive inference is perceived as philosophically problematic at all, the temptation to which most philosophers succumb is to offer too strong a justification: to say, for instance, that when we recognize the premises of a valid inference as true, we have thereby already recognized the truth of the conclusion.(Dummett, 1991, p. 195)

According to Hintikka, this can be described as a "scandal of deduction":

C.D. Broad has called the unsolved problems concerning induction a scandal of philosophy. It seems to me that in addition to this scandal of induction there is an equally disquieting scandal of deduction. Its urgency can be brought home to each of us by any clever freshman who asks, upon being told that deductive reasoning is "tautological" or "analytical" and that logical truths have no "empirical content" and cannot be used to make "factual assertions": in what other sense, then, does deductive reasoning give us new information? Is it not perfectly obvious there is some such sense, for what point would there otherwise be to logic and mathematics? (Hintikka, 1973, p. 222)

and, to quote Dummett again:

If that were correct, all that deductive inference could accomplish would be to render explicit knowledge that we already possessed: mathematics would be merely a matter of getting things down on paper, since, as soon as we had acknowledged the truth of the axioms of a mathematical theory, we should thereby know all the theorems. Obviously, this is nonsense: deductive inference has here been justified at the expense of its power to extend our knowledge and hence of any genuine utility.(Dummett, 1991, p. 195)

By trivializing deductive reasoning, the ordinary approach to semantic information gives rise to a new problem which appears to be worse than the one it set out to solve: if the conclusion of a deductive argument is always "contained" in the premises, why is deductive reasoning generally perceived as highly valuable epistemically? If all theorems are "contained" in the axioms of a theory, how is mathematical discovery possible at all?

In the following pages, we shall argue that there is a very reasonable sense in which classical deduction is actually informative, even at the propositional level, and hence that the scandal of deduction can be dissolved.

\footnotetext{
${ }^{2}$ For a recent reconstruction of this informational-analytical interpretation of classical deduction see (Corcoran, 1998).
}

DagostinoFloridi-kl.tex; 8/09/2008; 15:00; p.4 
To begin with, in Section 2 we shall briefly review three classic attempts to avoid the scandal, none of which turns out to be particularly satisfactory. The constructive part of this section will consist in showing both the evolution of the problem and how our strategy is comparable to the one adopted by Jaakko Hintikka, insofar as we shall also distinguish, as Hintikka does in (Hintikka, 1973), between degrees of informational depth. At the end of this section, we shall be left with a slightly revised version of the problem: how can the information carried by the conclusion of a propositional inference be entirely "contained" in the information carried by the premises, given that this information, according to a well-known conjecture in computational complexity, cannot be unpacked by means of a computationally feasible procedure? ${ }^{3}$

In Section 3, we shall investigate this problem against the background of the traditional tenet that logical reasoning is "analytical", examine the various senses in which the word "analytical" has been used in this connection, and lay down our general strategy for solving the problem via an informational-oriented approach.

In Sections 4 and 5, we shall critically examine two alternative approaches to classical semantics and show why they are unsuitable to provide a good basis for our sought-after informational approach to logical deduction.

Section 6 is the heart of our positive proposal. There we identify a new, stricter, sense in which classically valid inferences may be nonanalytical: when any proof of their validity essentially requires the (temporary) introduction of virtual information, ${ }^{4}$ namely information that is by no means contained in the premises, but must nevertheless be taken into consideration in order to obtain the conclusion. Analytical inferences, in this stricter sense, are exactly those that do not require any essential use of such virtual information. We argue that this stricter sense of "analytical" can be motivated, in the Kantian tradition, by the requirement that analytical reasoning should not be "augmentative": the train of thought that leads from the premises to the conclusion should not visit information states that lie outside the one defined by the premises. On the other hand, the use of virtual information is so entangled with the traditional semantical approaches (discussed in

\footnotetext{
3 This question, which is the background problem of the present paper, was put forward and discussed in (D'Agostino and Mondadori, 1999). Here we improve on that analysis and attempt to find a solution.

4 We are adapting here an expression from physics, where virtual particles are particle/antiparticle pairs which come into existence out of nothing and then rapidly annihilate without releasing energy. They populate the whole of space in large numbers but cannot be observed directly.
} 
Sections 4 and 5), that it appears to be inextricably intertwined with the meaning of the classical propositional operators.

So, in Section 7, we start to untie this knot and propose a redefinition of the logical operators which avoids any appeal to virtual information. We initially address the problem in terms of the "meaning as use" approach that, in logic, is traditionally associated with Gentzenstyle natural deduction systems and with the related idea that the meaning of the logical operators can be defined by specifying appropriate intelim (introduction and elimination) rules for them. We then propose an alternative to classical natural deduction which, though being compliant with all the strictest requirements for a meaningful and well-behaved deductive system that have emerged from the Gentzen tradition, is weaker than the standard one. Its intelim rules can be interpreted as characterizing "a" meaning of the logical operators we call it their informational meaning — which does not require any use of virtual information.

In Section 8, we show that deducibility in this natural deduction system is a computationally tractable problem, satisfies the subformula property and admits of an efficient normalization procedure, showing that the difficulties which motivate this paper disappear by waiving virtual information.

In Section 9, we address the same problem - that of characterizing the informational meaning of the logical operators - from a different and more "semantic" perspective, in terms of valuations. According to this approach, the meaning of an operator is given by specifying a set of constraints on admissible partial valuations which represent possible information states (rather than "possible worlds" which are, by way of contrast, represented by standard Boolean valuations).

This leads us, in Section 10, to define the notions of shallow information state and shallow information content, which are based on the informational meaning of the logical operators discussed in the previous sections. These notions can be contrasted to those of "possible world" and "semantic information", which are based on their classical meaning. Uninformative deductive reasoning, the truly "analytical" one, can now be identified with that kind of reasoning that does not increase the shallow information carried by the premises.

In Section 11, we address the problem of gradually retrieving the full deductive power of classical propositional logic by means of a bounded recursive use of virtual information. While the latter is banned from the basic "strictly analytical" deductive arguments, those which are truly uninformative, its incremental iterated use leads to more and more powerful deductive systems, which exhibit increasing levels of logical depth. Although these systems are all tractable, their growing compu- 
tational complexity approaches intractability as their deductive power approaches that of classical propositional logic. ${ }^{5}$ The underlying suggestion is that such incremental use of virtual information corresponds to increasing degrees of "synthetic" reasoning in classical propositional logic.

\section{Three classic attempts to avoid the scandal}

The standard attempt to sweep the scandal under a psychologistic rug is well-represented by Hempel's words:

It is typical of any purely logical deduction that the conclusion to which it leads simply re-asserts (a proper or improper) part of what has already been stated in the premises. Thus, to illustrate this point by a very elementary example, from the premise, "This figure is a right triangle", we can deduce the conclusion, "This figure is a triangle"; but this conclusion clearly reiterates part of the information already contained in the premise. [...] The same situation prevails in all other cases of logical deduction; and we may, therefore, say that logical deduction - which is the one and only method of mathematical proof - is a technique of conceptual analysis: it discloses what assertions are concealed in a given set of premises, and it makes us realize to what we committed ourselves in accepting those premises; but none of the results obtained by this technique ever goes by one iota beyond the information already contained in the initial assumptions. Since all mathematical proofs rest exclusively on logical deductions from certain postulates, it follows that a mathematical theorem, such as the Pythagorean theorem in geometry, asserts nothing that is objectively or theoretically new as compared with the postulates from which it is derived, although its content may well be psychologically new in the sense that we were not aware of its being implicitly contained in the postulates. (Hempel, 1945)

According to this view, the role of logical reasoning is that of helping us to bring out the full informational content of sentences, so that one can clearly see that the conclusion is contained in the premises by simple inspection. It is as if the premisses of a logical deduction were like compressed springs: they don't generate new information but merely store it and then release it again once they return to their original shape, namely once the deduction is fully laid down to include the conclusions.

\footnotetext{
${ }^{5}$ The idea of approximating classical propositional logic via a sequence of tractable subsystems has been pursued by several authors, with an entirely different motivation, in the context of automated reasoning. See the end of Section 11 for a brief discussion of the literature.
} 
For Wittgenstein, the whole problem was, needless to say, a pseudoproblem, arising from our use of an imperfect language. Given a "suitable notation", logical deduction could actually be reduced to the mere inspection of propositions:

When the truth of one proposition follows from the truth of others, we can see this from the structure of the propositions. (Tractatus, 5.13)

In a suitable notation we can in fact recognize the formal properties of propositions by mere inspection of the propositions themselves. (6.122).

Every tautology itself shows that it is a tautology. (6.127(b))

In accordance with Wittgenstein's idea, one could specify a procedure that translates sentences into a "perfect notation" that fully brings out the information they convey, for instance by computing the whole truthtable for the conditional which represents the inference. Such a table displays all the relevant possible worlds and allows one to distinguish immediately those that make a sentence true from those that make it false, the latter representing (collectively) the "semantic information" carried by the sentence. Once the translation has been performed, logical consequence can be recognized by "mere inspection" as being "clear and distinct" in a Cartesian sense. Following the truth-table example, in order to recognize the soundness of the inference one would only need to check whether the column corresponding to the conclusion contains " 1 " for each row where all the premises are given the value " 1 ". This clearly shows that the conclusion is true in all possible worlds where the premises are true; therefore the set of possible worlds excluded by the conclusion (that make the conclusion false) is included in the set of all possible worlds that are excluded by the conjunction of the premises (that make all the premises false).

Thus, if information could be fully unfolded by means of some mechanical procedure, the scandal of deduction could be avoided without appealing to psychologism. Sometimes we fail to immediately "see" that a conclusion is implicit in the premises because we express both in a concise notation, a sort of stenography that prevents us from fully recognizing the formal properties of propositions until we decode it into an adequate notation. Semantic information is a perfectly good way of specifying the information carried by a sentence with reference to an algorithmic procedure of translation.

Although this idea may seem (at first sight) to work well for propositional logic, one can easily see how it clashes with the undecidability of first-order logic. To put it with Hintikka:

$[\ldots]$ measures of information which are not effectively calculable are wellnigh absurd. What realistic use can there be for measures of information which are such that we in principle cannot always know (and cannot have 
a method of finding out) how much information we possess? One of the purposes the concept of information is calculated to serve is surely to enable us to review what we know (have information about) and what we do not know. Such a review is in principle impossible, however, if our measures of information are non-recursive.(Hintikka, 1973, p. 228)

At this point, it is useful to consider Hintikka's proposal, if only briefly. It consists in distinguishing between depth information and surface information. The former coincides with Carnap's and Bar-Hillel's semantic information and is a kind of potential information, which is non-computable and is not increased by deductive inference. By contrast, the latter is effectively computable and is increased by deductive inference. The growth of surface information is related to inference steps that introduce new individuals in the argument, which Hintikka describes as examples of the Kantian notion of "synthetic a priori" arguments. In Hintikka's approach, propositional logic turns out to be genuinely tautological or "analytical" in the sense of being entirely uninformative: for Boolean sentences surface and depth information coincide, so propositional inferences are really trivial, as witnessed by the existence of a mechanical decision procedure.

Hintikka's proposal has been variously criticized. ${ }^{6}$ One of its drawbacks is that it applies only to a restricted set of deductions in polyadic predicate calculus, and does not apply at all to deductions in monadic predicate calculus and propositional calculus. So we shall not follow Hintikka's detailed proposal regarding first-order logic. We shall, however, endorse his general strategy, based on a distinction between different degrees of informational depth. As will be shown presently, this is a fruitful way of tackling the question whether propositional logic really is informationally "tautological" or "analytical".

When Hintikka put forward his proposal, the impact of the research area known as "computational complexity" had not yet been fully appreciated. The distinction drawn within the domain of effective procedures between those that are "feasible", i.e. run in polynomial time, and those that are not, is widely accepted today. Within the domain of what is decidable, the attention is now focused on "tractable" problems, i.e. those that are solved by a feasible procedure. Tractable problems are regarded as the only problems that are solvable "in practice". On the other hand, there are problems that, although being algorithmically solvable in principle, are provably "intractable": the most efficient algorithm that solves them runs in superpolynomial (typically exponential) time.

\footnotetext{
${ }^{6}$ On this point, see the recent (Sequoiah-Grayson, 2008)
} 
A celebrated theorem, due to Stephen Cook ((Cook, 1971)), has shown that the tautology problem is computationally hard (NP-hard). This means that it belongs to a large class of difficult problems related to each other by a common computational fate: the existence of a feasible (polynomial time) algorithm to solve one of these problems would involve the existence of a similar procedure for all of them. Therefore, by a commonly accepted conjecture, the tautology problem is regarded as intractable.

The starting point of our investigation is that the (almost certain) intractability of classical propositional logic provides an argument against the idea that propositional inference is "tautological" or "analytical", in the sense clarified above. If classical propositional calculus is computationally intractable, it is simply false that "when the truth of one proposition follows from the truth of others, we can see this from the structure of the propositions" because, pace Wittgenstein, many nontrivial deductive structures are just too complex to unravel. It is not that we cannot see and explore the logical space in question, but that no mechanical procedure will ever have sufficient time to complete the full explorations required.

This objection is nearly as strong as Hintikka's argument leading to a similar rejection in the case of first-order logic, due to its undecidability. In the propositional case, the problem of unfolding semantic information, though being solvable by means of an effective procedure, is (most likely) intractable. The result is that Hintikka's claim can be reworded thus:

Measures of information which are not feasibly calculable are well-nigh absurd. What realistic use can there be for measures of information which are such that we in practice cannot always know (and cannot have a method of finding out) how much information we possess? One of the purposes the concept of information is calculated to serve is surely to enable us to review what we know (have information about) and what we do not know. Such a review is practically impossible, however, if our measures of information are intractable.

The reader who finds this argument plausible will agree that the solutions discussed above leave the scandal of deduction unmitigated even in the formerly "decent" field of propositional logic. The scandal can now be reformulated thus: how can the information carried by the conclusion of a propositional inference be "contained" in the information carried by the premises, given that this information cannot be unpacked by means of a feasible procedure? True, the existence of an effective procedure for computing semantic information in the case of propositional logic does make a difference with respect to the case of first-order logic. For it provides an objective and well-defined 
sense in which propositional logic is tautological. However, the main motivation for addressing this problem at all, namely the incompatibility between the alleged tautological character of logical reasoning and its "informational usefulness" remains unchanged. If propositional logic is informationally "trivial", why is the tautology problem so hard? And why are so many efforts made, both in theoretical and in applied computer science, to try and solve special cases of this allegedly trivial problem?

\section{What is analytical deduction?}

The characteristic of being "analytical", when used to qualify logical reasoning, may be interpreted in different senses. The most common are the following:

1. Semantic or meaning-theoretical. An inference is analytical when its soundness depends solely on the meaning of the logical operators which occur in its premises and conclusion. This sense of "analytical" usually comes with a theory of how the meaning of the logical operators is specified;

2. Informational. An inference is analytical when the information conveyed by its conclusion is "contained" in the information conveyed by its premises. In this second sense, "analytical" means simply "uninformative";

3. Syntactic. A formal deduction (a sequence of inference steps in a formal system of deduction) is analytical when it obeys the subformula principle. The exact formulation of this principle depends on the adopted formal system of deduction. However, the basic idea is that every formula occurring in the deduction must be a subformula of the final theorem to be proved. ${ }^{7}$ In Gentzen's words: "No concepts enter into the proof other than those contained in its final result, and their use was therefore essential to the achievement of that result"(Gentzen, 1935, p. 69) so that "the final result is, as it were, gradually built up from its constituent elements" (Gentzen, 1935, p.88).

\footnotetext{
7 Recall that a sentence $P$ is an immediate subformula of a sentence $P^{\prime}$ if and only if $P^{\prime}$ has one of the forms $P \vee Q, Q \vee P, P \rightarrow Q, Q \rightarrow P, P \wedge Q, Q \wedge P$, $\neg P$. A sentence $P$ is a subformula of a sentence $P^{\prime}$ if and only if there is a sequence $P_{1}, \ldots, P_{n}$, with $n \geq 1$, such that (i) $P_{1}=P^{\prime}$, (ii) $P_{n}=P$, and (iii) $P_{i+1}$ is an immediate subformula of $P_{i}$, for $i=0,1, \ldots, n-1$. Notice that, according to this definition, $P$ is a subformula of $P$ itself. A proper subformula of $P$ is any subformula of $P$ which is different from $P$ itself.
} 
While the third, syntactic sense is utterly clear and relies on the technical notion of "subformula" or "constituent" of a sentence, the first sense depends on a more precise formulation of the adopted theory of meaning for the logical operators, and the second on a clarification of the notion of information content and of what it means that some amount of information is "contained" in another. Despite this residual vagueness, the semantic and informational senses are closely related to each other and to the syntactic one. For, it seems natural to assume that an inference carries no information if and only if its conclusion can be "seen" as true, whenever its premises are true, by simply looking at the definitions of the logical words occurring in the sentences. In this sense, one could say that analytical inferences are those which are recognized as sound via steps which are all "explicative", that is, descending immediately from the meaning of the logical operators, and not "augmentative", involving some intuition that goes beyond this meaning. ${ }^{8}$ From this point of view, it seems reasonable to require the semantic and informational senses to be co-extensional:

\section{All inferences that are analytical in the semantic sense should} be analytical also in the informational sense and vice versa.

Moreover, if an inference is analytical in the semantic sense, then it should be possible to prove its soundness by means of some formal deduction which is analytical in the syntactic sense as well. This expectation seems further justified if one adopts a "molecular" view of language, that is "any view in which sentences carry a content which belongs to them in accordance with the way they are compounded out of their constituents, independently of other sentences of the language not involving those constituents". ${ }^{9}$ So, a deductive inference which is analytical in the semantic sense, should not require the consideration

\footnotetext{
8 "Analytical judgements (affirmative) are therefore those in which the connection of the predicate with the subject is cogitated through identity; those in which this connexion is cogitated without identity, are called synthetical judgements. The former may be called explicative, the latter augmentative judgements; because the former add in the predicate nothing to the conception of the subject, but only analyse it into its constituent conceptions, which were thought already in the subject, although in a confused manner; the latter add to our conceptions of the subject a predicate which was not contained in it, and which no analysis could ever have discovered therein." Immanuel Kant, Critique of Pure Reason, translated by J.M.D. Meiklejohn, Henry G. Bohn, London 1855. Mutatis mutandis, one could say that an inference is analytical if it adds in the conclusion nothing to the information contained in the premises, but only analyses it in its constituent pieces of information, which were thought already in the premises, although in a confused manner. The confusion vanishes once the meaning of the logical operators is properly explicated.

9 (Dummett, 1978), p. 222.
} 
of any linguistic expression which is not a constituent of its premises or of its conclusion. Therefore, it also seems natural to require that:

All inferences that are analytical in the semantic-informational sense should be provable by means of formal deductions which are analytical in the syntactic sense, that is, such that they obey the subformula principle.

Let us then say that a $\operatorname{logic}^{10} L$ is analytical if, whenever $\Gamma \vdash_{L} P$ for some set of sentences $\Gamma$ and some sentence $P$, the inference from $\Gamma$ to $P$ is analytical both in the semantic and the informational sense. Given our assumption (4) above, we should also expect that for any analytical logic $L$, there exists a formal system of deduction $S$ which is sound and complete for $L$ and such that, whenever $\Gamma \vdash_{L} P$, there is an $S$-deduction of $P$ from $\Gamma$ which is analytical in the syntactic sense, i.e. that enjoys the subformula property.

It may seem, at first sight, that Boolean logic meets all our requirements for such an analytical logical system:

1. all the sound inferences of Boolean logic are analytical in the semantic sense, once the meaning of the logical operators is explicated via the usual truth-table semantics;

2. all the sound inferences of Boolean logic are analytical in the informational sense, once the notion of information content of a sentence is explicated via the theory of semantic information;

3. given classical semantics and the classical notion of semantic information, the semantic and informational sense of "analytical" are co-extensional, in accordance with our requirement (3) above;

4. there are proof-theoretical formulations of Boolean logic (e.g. the truth-table method, Gentzen's sequent calculus $L K$ or Smullyan's analytical tableaux) in which all sound inferences can be proved by means of formal deductions which are analytical in the syntactic sense, in accordance with our requirement (4) above.

However, as argued in the introduction, the computational "hardness" of Boolean reasoning spoils this rosy picture. According to the widely accepted conjecture that $P \neq N P$, most probably there is no practical decision procedure to establish whether the semantic information carried by the conclusion of an inference is contained in that carried by its premises. It seems, therefore, paradoxical to claim that we are unable to decide, in practice, whether the conclusion carries

10 Following Tarski, we identify a logic $L$ with a consequence relation, that is, a relation $\vdash_{L}$ between sets of formulas and formulas satisfying reflexivity $\left(\Gamma \cup\{P\} \vdash_{L} P\right.$ for every set of formulas $\Gamma)$, monotonicity $\left(\Gamma \vdash_{L} P\right.$ implies $\Gamma \cup \Delta \vdash_{L} P$, for all $\left.\Gamma, \Delta\right)$, and transitivity $\left(\Gamma \vdash_{L} P\right.$ and $\Delta \cup\{P\} \vdash_{L} Q$ imply $\left.\Gamma \cup \Delta \vdash_{L} Q\right)$. The monotonicity condition is also called weakening, and the transitivity condition is also called cut.

DagostinoFloridi-kl.tex; 8/09/2008; 15:00; p.13 
any new information. Even worse, according to the (almost as widely) accepted conjecture that $N P \neq c o-N P$, the length of formal proofs in propositional logic cannot be efficiently bounded, and so we may even be unable, in practice, to show that the information content of the conclusion is contained in the information content of the premises. According to this view, the information content of a sentence should be something that "is there", but often cannot be feasibly expressed or communicated. This appears to be rather distant from what is commonly intended by "information" and suggests that the standard notion of information content, defined in terms of semantic information is, in some sense, too strong.

At this point, we are left with the following problem:

PROBLEM 1. Is there a sensible, weaker, definition of the information content of a sentence - let us call it "shallow information content" - such that it is always feasibly recognizable whether the information carried by the conclusion is contained in the information carried by the premises?

The expression "sensible" indicates that we are not looking for any weaker notion of information content that solves our Problem $1 .^{11} \mathrm{In}$ view of our assumption (3) above, a solution to this problem must consist in a weaker notion of information content, which can be paired with a correspondingly weaker characterization of the meaning of the logical operators, in such a way that, if this weaker characterization is adopted, the associated semantic sense of "analytical" would still be co-extensional with the informational sense.

Thus, our new problem is: what is wrong with classical semantics? What is it that makes it unsuitable to define a feasible notion of information content? To put it positively:

PROBLEM 2. Is there a sensible semantics for the logical operators of Boolean logic which is (i) weaker than the standard truth-table semantics, and (ii) better suited to define a corresponding feasible notion of information content?

This problem, namely the semantic characterization of tractable inference, has received a good deal of attention in computer science circles, but it has never, to the best of our knowledge, been addressed from the philosophical vantage point that we take in this paper. In Sections 69 , we shall propose a solution to Problem 2 which will be key to our

\footnotetext{
11 Otherwise, this could be trivially achieved in a variety of ways, for instance, by simply assuming that the information content of a sentence consists in the set containing the sentence itself, and that the corresponding analytical inferences of Boolean logic are only the inferences $\Gamma \vdash P$ such that $P \in \Gamma$.
} 
characterization of a feasible notion of information content. Problem 1 will then be solved in section 10 .

Let us first examine why more conventional semantic approaches are not suitable for this purpose.

\section{Why classical truth-conditional semantics is unsuitable}

What is a "sensible" semantics for the logical operators? Classical logicians have a straightforward answer to this question: the time-honoured semantics based on the truth-table definitions. The meaning of each logical operator $\sharp$ is explicated by providing necessary and sufficient conditions under which a sentence containing $\sharp$ as its main operator is, respectively, true and false in a given state of affairs $w$.

C1 $\neg P$ is true in $w$ if and only if $P$ is false in $w$;

C2 $P \wedge Q$ is true in $w$ if and only if $P$ is true in $w$ and $Q$ is true in $w$;

C3 $P \vee Q$ is true in $w$ if and only if $P$ is true in $w$ or $Q$ is true in $w$;

C4 $P \rightarrow Q$ is true in $w$ if and only if $P$ is false in $w$ or $Q$ is true in $w$;

C5 $\neg P$ is false in $w$ if and only if $P$ is true in $w$;

C6 $P \wedge Q$ is false in $w$ if and only if $P$ is false in $w$ or $Q$ is false in $w$;

C7 $P \vee Q$ is false in $w$ if and only if $P$ is false and $Q$ is false in $w$;

C8 $P \rightarrow Q$ is false in $w$ if and only if $P$ is true and $Q$ is false in $w$.

Such conditions provide an explanation of the meaning of the logical operators in terms of the two central notions of truth and falsity, which are assumed as understood. It is regarded as essential to understanding of these notions that they obey the classical principles of bivalence (each sentence, in a given state of affairs, is either determinately true or determinately false) and non-contradiction (no sentence can be at the same time true and false in the same state of affairs). Both principles can be concisely expressed by assuming that a sentence is false if and only if it is not true. Observe that, if the principle of bivalence is accepted as part of the classical meaning of "true" and "false", clauses C5-C8 become redundant since they can be derived from clauses $\mathrm{C} 1-\mathrm{C} 4$.

This approach has been severely criticized as too "metaphysical". As Weir puts it, for example:

DagostinoFloridi-kl.tex; 8/09/2008; 15:00; p.15 
[Classical semantics] has come under a great deal of attack, especially from those who subscribe to the Wittgensteinian slogan that meaning is use and interpret it as requiring that all ingredients of meaning can be made manifest in our use of sentences, especially in teaching or communicating their senses, for it is often claimed that classical bivalent semantics, in ascribing truth-values to sentences regardless of whether these values are discoverable, violates this requirement.(Weir, 1986, p. 459)

A less "metaphysical" approach might consist in replacing classical truth and falsity, as central notions of the theory of meaning, with other, more accessible ones. Since we are looking for a semantic characterization of the logical operators more in tune with a feasible notion of information content, it seems natural to explore the possibility of specifying the meaning of the logical operators in terms of central notions of informational nature. Suppose, for example, that we replace the expressions " $P$ is true in the state of affairs $w$ " and " $P$ is false in the state of affairs $w$ " in clauses $\mathrm{C} 1-\mathrm{C} 8$ with, respectively, "the piece of information ' $P$ is true' belongs to the state of information $S$ " and "the piece of information ' $P$ is false' belongs to the state of information $S$ ". It is obvious that this move immediately implies that the classical Principle of Bivalence should be abandoned: there is no reason to assume that, given an arbitrary sentence $P$ and a state of information $S$, either the piece of information " $P$ is true" or the piece of information " $P$ is false" should belong to $S$.

Moreover, it is apparent that some of the clauses that define the meaning of the logical operators also cease to be intuitively sound. Consider, for instance, clause $\mathrm{C}$ 3: if a piece of information " $P \vee Q$ is true" belongs to a given information state $S$, this by no means implies that either the piece of information " $P$ is true" belongs to $S$ or the piece of information " $Q$ is true" belongs to $S$. Under the commonsense notion of information, it is perfectly reasonable that we hold the information that $P \vee Q$ is true without holding either the information that $P$ is true or the information that $Q$ is true. Indeed, this kind of irreducible disjunctive information is essential in most database applications. Similar considerations hold for clause C6: holding the information that $P \wedge Q$ is false does not imply either holding the information that $P$ is false or holding the information that $Q$ is false.

Hence, some of the truth-table conditions that provide the classical definitions of the logical operators do not survive such an informational reinterpretation. This suggests that, from this point of view, the operators are somehow overdefined and this in turn leaves us with the problem of whether the standard necessary and sufficient conditions can be reformulated so as to provide a coherent and intuitively sound "informational semantics" for the logical operators. 
Before addressing this problem, let us examine an important alternative conception regarding the meaning of the logical operators, which seems more attuned to the "meaning as use" approach usually ascribed to Wittgenstein.

\section{Why classical intelim semantics is unsuitable}

The semantic conception discussed in this section can be traced back to the way in which Gentzen presented his "natural deduction" systems. It consists in defining the logical operators directly in terms of the basic rules that govern their use in deductive reasoning. From this point of view, the meaning of a logical operator is defined by a set of primitive logical rules that completely determine the role it plays in any deductive argument. Typically, such primitive rules for an operator $\sharp$ must specify both the immediate conditions, under which a sentence containing $\sharp$ as the main operator can be introduced into a deductive argument (introduction rules for $\sharp$ ), and the consequences that can be immediately drawn from such a sentence (elimination rules for $\sharp$ ). We call this approach intelim semantics for the logical operators. Gentzen's natural deduction system $N J$ for intuitionistic logic provides a paradigmatic example. ${ }^{12}$ The inference rules in such a natural deduction system are best formulated in terms of sequents, namely expressions of the form $\Gamma \Longrightarrow P$ where $\Gamma$ is a finite set of sentences (the assumptions) and $P$ is a single sentence (the conclusion), whose standard reading is "we have a deduction of $P$ from the assumptions in $\Gamma$ ". Intelim rules have sequents as premises and as conclusions. For instance, the introduction rule for $\wedge$ is:

$$
\frac{\Gamma \Longrightarrow P \quad \Delta \Longrightarrow Q}{\Gamma, \Delta \Longrightarrow P \wedge Q}
$$

\footnotetext{
12 Gentzen himself suggested that the rules of this system could be taken as definitions of the logical operators. Indeed, he proposed that the introduction rules would be sufficient for this purpose and that the elimination rules could be ultimately "justified" in terms of the introduction rules, and this idea was later refined into criteria of admissibility for putative definitions of the logical operators that culminated in Prawitz's inversion principle: no information can be obtained from applying an elimination rule to a sentence $P$ that would not have already been available if $P$ had been obtained by means of an introduction rule. For instance, by applying the elimination rules for $\wedge$ to the sentence $P \wedge Q$ we can obtain $P$ and $Q$, both of which would have already been available if $P \wedge Q$ had been obtained by means of the standard introduction rule for $\wedge$. It is exactly this "harmony" between introduction and elimination rules that guarantees that the deductive system as a whole is syntactically analytic, i.e. that it enjoys the subformula property.
}

DagostinoFloridi-kl.tex; 8/09/2008; 15:00; p.17 
where $\Gamma, \Delta$ means $\Gamma \cup \Delta$, the two sequents above the horizontal line are the premises, and the sequent below the line is the conclusion. The rule says: "if we have a deduction of $P$ from the assumptions in $\Gamma$ and we have also a deduction of $Q$ from the assumptions in $\Delta$, then we ipso facto have a deduction of $P \wedge Q$ from the assumptions in $\Gamma \cup \Delta$."

A typical feature of natural deduction is that some of the rules allow for the discharge of assumptions. For instance, the standard introduction rule for $\rightarrow$ has the form:

$$
\frac{\Gamma, P \Longrightarrow Q}{\Gamma \Longrightarrow P \rightarrow Q}
$$

where $\Gamma, P$ is a simplified form for $\Gamma \cup\{P\}$, and the standard elimination rule for $\vee$ has the form:

$$
\frac{\Gamma \Longrightarrow P \vee Q \quad \Delta, P \Longrightarrow R \quad \Lambda, Q \Longrightarrow R}{\Gamma, \Delta, \Lambda \Longrightarrow R .}
$$

In these rules, some of the assumptions which occur in the premisesequents (namely $P$ in the first rule, and $P$ and $Q$ in the second) do not occur in the conclusion sequent. They are regarded as "provisional" or "virtual" assumptions, which are "discharged" by the application of the rule. The reader is referred to any good exposition of Gentzen's natural deduction (such as (Tennant, 1990)) for the details.

This approach seems to work very well with intuitionistic logic, but there is a problem when it is transposed into the context of classical logic: it is impossible to find a system of intelim rules which is (i) sound and complete for classical propositional logic and (ii) analytical in the syntactic sense, i.e. such that it enjoys the subformula property. ${ }^{13}$ Gentzen's solution to this problem was more technical than philosophical, and consisted in introducing multi-conclusion sequents, namely expressions of the form $\Gamma \Longrightarrow \Delta$ where both $\Gamma$ and $\Delta$ are finite sets of sentences, as the basic proof-theoretical units. ${ }^{14}$ However, this can hardly be considered as a satisfactory solution. As Dummett puts it:

A sequent of the form $A \Longrightarrow B, C$ cannot be explained by saying "if you have asserted $A$, you may with equal right assert either $B$ or $C$ ", for that would imply that you can assert either one at your choice; and the formulation, "If you have asserted $A$, then either you may assert $B$ or you

\footnotetext{
13 On this point see, for instance, (Leblanc, 1966).

14 Gentzen developed this idea into a new calculus, the sequent calculus, which is quite different from the original natural deduction setting. Cellucci has shown how a form of multi-conclusion natural deduction can be better suited for the analysis of the logical operators. On this point see (Cellucci, 1988). For a general discussion of various formulations of classical natural deduction see also (Cellucci, 1995).
}

DagostinoFloridi-kl.tex; 8/09/2008; 15:00; p.18 
may assert $C^{\prime \prime}$, does not entitle you to make any further assertion until you learn which of them you may assert. ${ }^{15}$

A better solution consists, in the case of classical propositional logic, to shift from sentences to "signed" sentences as assumptions and conclusions of sequents. ${ }^{16}$ A signed sentence is an expression of the form t $P$ or $\mathbf{f} P$, where $P$ is a sentence of a standard propositional language. Note that the signs $\mathbf{t}$ and $\mathbf{f}$ are by no means to be interpreted as new logical operators, in that they cannot be used inside a sentence, nor can they be iterated. They can only be used to prefix a sentence; their intuitive meaning is that the prefixed sentence is evaluated as "true" and "false" respectively. If signed formulas become the basic units of logical reasoning, and inference rules are, accordingly, formulated in terms of sequents of signed formulas, then several natural deduction systems can be presented which enjoy the full subformula property. The reason is that all the troubles that arise when one tries to explain classical negation in terms of the notion of "proof" - intended as the central notion of semantic theory by means of which the meaning of the logical operators is to be specified ${ }^{17}$ — disappear when the classical notions of truth and falsity replace it in this role. ${ }^{18}$

In what follows, we shall use $\varphi, \psi$, etc. to denote signed formulas, and $X, Y$, etc. to denote finite sets of signed formulas. We shall also denote by $\bar{\varphi}$ the conjugate of $\varphi$, that is, the signed formula $\mathbf{t} P$ if $\varphi=\mathbf{f} P$ and the signed formula $\mathbf{f} P$ if $\varphi=\mathbf{t} P$. A rather elegant natural deduction system of this kind - which is closely related to Smullyan's analytic tableaux, to Gentzen's sequent calculus, to Smullyan's "analytic natural deduction" (Smullyan, 1965), and to the systems EN and EN* discussed in (Bendall, 1978) - consists of the sequent rules illustrated in Table I. ${ }^{19}$ What happens if we apply the same informational reinterpretation to this natural deduction system that we applied in the previous section to the classical truth-tables? That is, if we reinterpret

15 (Dummett, 1991), p. 187.

16 For the background motivations of this approach, see (Bendall, 1978).

17 On the role of such a "central notion", see (Dummett, 1991), especially pp. 32$33,62-64$ and 317 .

18 Accordingly, the very notion of subformula must be modified: a subformula of a signed formula $\mathbf{s} P$, where $\mathbf{s}$ is $\mathbf{t}$ or $\mathbf{f}$, is any signed formula $\mathbf{s} Q$ where $Q$ is a subformula of $P$.

19 Actually, half of the operational rules of this system are redundant. The structural rule $R A$ allows one to derive the introduction rules from the elimination rules and vice versa. However, this redundant presentation is useful in order to highlight the connections between this system and standard natural deduction; moreover, in the following pages it will help us to compare this system with an alternative natural deduction system in which the classical reductio ad absurdum rule is not allowed and therefore introduction and elimination rules are not derivable from each other. 
Table I. A system of natural deduction with signed formulas for classical propositional logic.

$$
\begin{aligned}
& R \quad X \Longrightarrow \varphi \text { provided that } \varphi \in X \\
& W \quad X \Longrightarrow \varphi / X^{\prime} \Longrightarrow \varphi \text { provided that } X \subseteq X^{\prime} \\
& R A \quad X, \varphi \Longrightarrow \psi ; X, \varphi \Longrightarrow \bar{\psi} / X \Longrightarrow \bar{\varphi} \\
& \mathbf{t} \wedge I \quad X \Longrightarrow \mathbf{t} P ; X \Longrightarrow \mathbf{t} Q / X \Longrightarrow \mathbf{t} P \wedge Q \\
& \mathbf{t} \wedge E \quad X \Longrightarrow \mathbf{t} P \wedge Q / X \Longrightarrow \mathbf{t} P \\
& X \Longrightarrow \mathbf{t} P \wedge Q / X \Longrightarrow \mathbf{t} Q \\
& \mathbf{t} \vee I \quad X \Longrightarrow \mathbf{t} P / X \Longrightarrow \mathbf{t} P \vee Q \\
& X \Longrightarrow \mathbf{t} Q / X \Longrightarrow \mathbf{t} P \vee Q \\
& \mathbf{t} \vee E \quad X \Longrightarrow \mathbf{t} P \vee Q ; X, \mathbf{t} P \Longrightarrow \varphi ; X, \mathbf{t} Q \Longrightarrow \varphi / X \Longrightarrow \varphi \\
& \mathbf{t} \rightarrow I \quad X \Longrightarrow \mathbf{f} / X \Longrightarrow \mathbf{t} P \rightarrow Q \\
& X \Longrightarrow \mathbf{t} Q / X \Longrightarrow \mathbf{t} P \rightarrow Q \\
& \mathbf{t} \rightarrow E \quad X \Longrightarrow \mathbf{t} P \rightarrow Q ; X, \mathbf{f} P \Longrightarrow \varphi ; X, \mathbf{t} Q \Longrightarrow \varphi / X \Longrightarrow \varphi \\
& \mathbf{t} \neg I \quad X \Longrightarrow \mathbf{f} P / X \Longrightarrow \mathbf{t} \neg P \\
& \mathbf{t} \neg E \quad X \Longrightarrow \mathbf{t} \neg P / X \Longrightarrow \mathbf{f} P \\
& \mathbf{f} \wedge I \quad X \Longrightarrow \mathbf{f} P / X \Longrightarrow \mathbf{f} P \wedge Q \\
& X \Longrightarrow \mathbf{f} Q / X \Longrightarrow \mathbf{f} P \wedge Q \\
& \mathbf{f} \wedge E \quad X \Longrightarrow \mathbf{f} P \wedge Q ; X, \mathbf{f} P \Longrightarrow \varphi ; X, \mathbf{f} Q \Longrightarrow \varphi / X \Longrightarrow \varphi \\
& \mathbf{f} \vee I \quad X \Longrightarrow \mathbf{f} P ; X \Longrightarrow \mathbf{f} Q / X \Longrightarrow \mathbf{f} P \vee Q \\
& \mathbf{f} \vee E \quad X \Longrightarrow \mathbf{f} P \vee Q / X \Longrightarrow \mathbf{f} P \\
& X \Longrightarrow \mathbf{f} P \vee Q / X \Longrightarrow \mathbf{f} Q \\
& \mathbf{f} \rightarrow I \quad X \Longrightarrow \mathbf{t} P ; X \Longrightarrow \mathbf{f} Q / X \Longrightarrow \mathbf{f} P \rightarrow Q \\
& \mathbf{f} \rightarrow E \quad X \Longrightarrow \mathbf{f} P \rightarrow Q / X \Longrightarrow \mathbf{t} P \\
& X \Longrightarrow \mathbf{f} P \rightarrow Q / X \Longrightarrow \mathbf{f} Q \\
& \mathbf{f} \neg I \quad X \Longrightarrow \mathbf{t} P / X \Longrightarrow \mathbf{f} \neg P \\
& \mathbf{f} \neg E \quad X \Longrightarrow \mathbf{f} \neg P / X \Longrightarrow \mathbf{t} P
\end{aligned}
$$

t $P$ as "the piece of information ' $P$ is true' belongs to the current state of information" and $\mathbf{f} P$ as "the piece of information ' $P$ is false' belongs to the current state of information"?

The rule $R A$, though closely related to the Principle of Bivalence, at first sight does not seem to be subject to the same criticism. We may find it acceptable that, if a new piece of information $\varphi$ is added 
to our information state, represented by the $X$ in the antecedent of the premises, and is thereafter discovered to be inconsistent with it, then we are entitled to say that the conjugate of $\varphi$ is "implicitly" contained in our current information state (under the assumption that the latter is consistent). Even the operational rules seem to survive the criticism we addressed before to their truth-table counterparts. Consider, for example, the rule $\mathbf{t} \vee E$. It says that, whenever (i) the piece of information " $P \vee Q$ is true" belongs to our current information state $S$, and (ii.a) expanding $S$ with the new piece of information " $P$ is true" or (ii.b) expanding $S$ with the new piece of information " $Q$ is true", leads in either case to a state of information that "contains" a piece of information $\varphi$, then we are entitled to say that $\varphi$ is "implicitly" contained in the current information state, by the very meaning of the logical word "V". This may be intuitively acceptable provided one makes the non-trivial assumption that it is always possible, from a state of information containing " $P \vee Q$ is true", to reach both a state of information containing " $P$ is true" and a state of information containing " $Q$ is true". Under this assumption, the rule explains part of the meaning of $\vee$ by considering "virtual" information states which may be reachable from the current one, though being definitely richer than it. However, in both such virtual information states there is a piece of information which is not contained in the current information state, in any possible sense of the word "contained". A similar comment also applies to the $R A$ rule and to all the "discharge rules" of the natural deduction system presented in Table I, as well as of any known (single-conclusioned) natural deduction system.

Our discussion in this section suggests that the meaning of the logical operators may be somehow overdefined by the standard intelim rules. Is their typical appeal to virtual information really necessary? Is it not possible to assign them a shallower meaning by means of intelim rules that involve only the current information state, with no need for a virtual tour of its potential expansions?

\section{A fourth sense of "analytical": no virtual information}

In his proof of Proposition 4 in the the first book of the Elements, the so-called "first congruence theorem on triangles", Euclid makes use of a method of proof which is sometimes called "superposition". Although Euclid's use of this method is not entirely obvious, according to many interpreters it consisted in actually moving one triangle over the other so as to cover it through a continuous motion. Now, it has been correctly observed that such a method would not be applicable to two triangles 
which are a mirror image of each other without moving one of them outside the plane. So, this method of proof would not be available if one, like the inhabitants of Abbott's Flatland, had no conception of a tridimensional space. The information carried by the conclusion is actually contained in the information carried by the premises only for the inhabitants of our familiar tridimensional space. Flatlandians have to make a special effort to go (temporarily) beyond what is given to them, namely beyond their bidimensional universe, in order to "see" that the conclusion is contained in the premises. They have to travel through a space that, for them, is entirely "virtual". Although this can be perfectly conceived and achieved, the reasoning involved could hardly be described as "analytical". Rather than being merely "explicative", it appears to be considerably "augmentative" (see footnote 8) exactly in Kant's sense. We argue that a similar augmentative process is involved when the natural deduction rules that make use of virtual information, namely those which are usually called "discharge rules", are applied. The reasoning agent who applies these rules has to make an effort to go (temporarily) beyond the information which is actually given to her, use some "virtual" information and then come back. This stepping out and in again of the given informational space is what makes the informativeness of classical propositional logic so invisible and yet present.

All this strongly suggests a fourth sense in which a logical inference can be "analytical". We shall call it the strict informational sense. An inference is analytical in a strict informational sense (or strictly analytical, for short) when the conclusion can be deduced from the premises without making use of any virtual information, i.e. when every sentence occurring in the proof belongs implicitly to any information state containing the information carried by the premises. Clearly, none of the discharge rules of typical natural deduction systems are analytical in this strict informational sense. In the context of formal deduction systems based on sequents of signed formulas, such as the one presented in Table I, this precisely means that no inference step is allowed in which the antecedent of some of the premises contains a signed formula which is not deducible from the signed formulas in the antecedent of the conclusion. In our example, the rules which turn out not to be analytical in the strict informational sense, and which we therefore call "augmentative", are exactly $R A, \mathbf{t} \vee E, \mathbf{t} \rightarrow E$ and $\mathbf{f} \wedge E$. 


\section{The informational meaning of the logical operators}

How can we adjust the system in Table I so as to make it analytical in the strict informational sense? Just removing the augmentative rules from the system would not be a satisfactory solution. For the result would not be a logical system in any acceptable sense of the word, even if it would still satisfy Tarski's definition of a consequence relation. ${ }^{20}$ First, observe that the removal of $R A$ makes the introduction and the elimination rules logically independent of each other, that is, the introduction rules can no longer be derived from the elimination rules, nor can the latter be derived from the former. As a result, the further removal of $\mathbf{t} \vee E$, of $\mathbf{t} \rightarrow E$ and of $\mathbf{f} \wedge E$ would leave the meaning of $\vee, \rightarrow$ and $\wedge$ only partially defined, so we could no longer claim that the rules provide a definition of the meaning of the logical operators. Second, the resulting system would be extremely weak. It would prevent us from deducing conclusions that are intuitively contained in the premises even in the strict informational sense introduced above. For instance, consider a typical disjunctive syllogism in which, from the information that a disjunction is true and one of the two disjuncts is false, we deduce that the other disjunct is true. This basic inference principle would no longer be derivable in such a truncated system. Intuitively, however, it is a strictly analytical inference principle, because we do not need any virtual information to establish that the conclusion is contained in the premises. This is a kind of reasoning "by exclusion" which may be explained in terms of virtual information, but may as well be directly regarded as part of the definition of $\vee$. The corresponding sequent rules, namely:

$$
\frac{X \Longrightarrow \mathbf{t} P \vee Q \quad X \Longrightarrow \mathbf{f} P}{X \Longrightarrow \mathbf{t} Q} \text { and } \frac{X \Longrightarrow \mathbf{t} P \vee Q \quad X \Longrightarrow \mathbf{f} Q}{X \Longrightarrow \mathbf{t} P}
$$

are analytical in the strict informational sense explained above and could replace the augmentative rule $\mathbf{t} \vee E$. The same holds true for the familiar modus ponens and modus tollens - allowing us, respectively, to deduce $\mathbf{t} Q$ from $\mathbf{t} P \rightarrow Q$ and $\mathbf{t} P$, and $\mathbf{f} P$ from $\mathbf{t} P \rightarrow Q$ and f $Q$ - which could not be derived in the truncated system. Again, the corresponding sequent rules, namely:

$$
\frac{X \Longrightarrow \mathbf{t} P \rightarrow Q \quad X \Longrightarrow \mathbf{t} P}{X \Longrightarrow \mathbf{t} Q} \text { and } \frac{X \Longrightarrow \mathbf{t} P \rightarrow Q \quad X \Longrightarrow \mathbf{f} Q}{X \Longrightarrow \mathbf{f} P}
$$

are analytical in the strict informational sense and could directly be taken as contributing to the definition of $\rightarrow$, replacing the augmentative

20 See fn. 10 above. 
rule $\mathbf{t} \rightarrow E$. Similarly, the strictly analytical rules:

$$
\frac{X \Longrightarrow \mathbf{f} P \wedge Q \quad X \Longrightarrow \mathbf{t} P}{X \Longrightarrow \mathbf{f} Q} \text { and } \frac{X \Longrightarrow \mathbf{f} P \wedge Q \quad X \Longrightarrow \mathbf{t} Q}{X \Longrightarrow \mathbf{f} P}
$$

could replace the augmentative rule $\mathbf{f} \wedge E$.

If the augmentative operational rules of Table I are put through such a "slimming" process and the augmentative structural rule $R A$ is removed, the result is a streamlined natural deduction system with no virtual information (no "discharge rules"), which is summarized in Table II.

Observe that:

1. all the rules are intuitively sound under the informational interpretation of the signs $\mathbf{t}$ and $\mathbf{f}$ discussed above;

2. the system contains both introduction and elimination rules for each logical operator;

3 . the elimination rules cannot be derived from the introduction rules, but can be justified by them in terms of Prawitz's inversion principle ${ }^{21}$ indeed, though they are not the strongest elimination rules that can be so justified, it can be shown that they are the strongest ones which are strictly analytical, i.e. analytical in the strict informational sense.

The intelim system presented in Table II, therefore, is the strongest intelim system that can be expressed in terms of rules that are analytical in the strict informational sense. In accordance with the natural deduction tradition, we may take its operational rules as defining "a" meaning of the logical operators, which is clearly weaker than their classical meaning. It is expressed in terms of central notions of informational (rather than metaphysical) nature, via the reinterpretation of the signs $\mathbf{t}$ and $\mathbf{f}$ discussed above, and we may therefore call it the informational meaning of the logical operators.

Note that, since there are no discharge rules, the sequent notation becomes completely unnecessary. In fact, a more straightforward and concise presentation of this logical system is given by means of intelim rules with signed formulas, rather than sequents of signed formulas, as premises and conclusions (see Tables III and IV). In the two-premise elimination rules, we call major premise the one containing the logical operator, and minor premise the other one. An intelim sequence based on a set of signed formulas $X$ is a sequence $\varphi_{1}, \ldots, \varphi_{n}$ of signed formulas such that each element $\varphi_{i}$ of the sequence is either (i) an element of $X$, or (ii) exemplifies the conclusion of one of the intelim rules in Tables III

\footnotetext{
21 On this point see (D'Agostino, 2005) which contains a discussion of these natural deduction rules in their unsigned version.
}

DagostinoFloridi-kl.tex; 8/09/2008; 15:00; p.24 
Table II. A system of intelim rules which are all analytical in the strict informational sense.

$$
\begin{aligned}
& R \quad X \Longrightarrow \varphi \text { provided that } \varphi \in X \\
& W \quad X \Longrightarrow \varphi / X^{\prime} \Longrightarrow \varphi \text { provided that } X \subseteq X^{\prime} \\
& \mathbf{t} \wedge I \quad X \Longrightarrow \mathbf{t} P ; X \Longrightarrow \mathbf{t} Q / X \Longrightarrow \mathbf{t} P \wedge Q \\
& \mathbf{t} \wedge E \quad X \Longrightarrow \mathbf{t} P \wedge Q / X \Longrightarrow \mathbf{t} P \\
& X \Longrightarrow \mathbf{t} P \wedge Q / X \Longrightarrow \mathbf{t} Q \\
& \mathbf{t} \vee I \quad X \Longrightarrow \mathbf{t} P / X \Longrightarrow \mathbf{t} P \vee Q \\
& X \Longrightarrow \mathbf{t} Q / X \Longrightarrow \mathbf{t} P \vee Q \\
& \mathbf{t} \vee E \quad X \Longrightarrow \mathbf{t} P \vee Q ; X \Longrightarrow \mathbf{f} P / X \Longrightarrow \mathbf{t} Q \\
& X \Longrightarrow \mathbf{t} P \vee Q ; X \Longrightarrow \mathbf{f} P / X \Longrightarrow \mathbf{t} Q \\
& \mathbf{t} \rightarrow I \quad X \Longrightarrow \mathbf{f} P / X \Longrightarrow \mathbf{t} P \rightarrow Q \\
& X \Longrightarrow \mathbf{t} Q / X \Longrightarrow \mathbf{t} P \rightarrow Q \\
& \mathbf{t} \rightarrow E \quad X \Longrightarrow \mathbf{t} P \rightarrow Q ; X \Longrightarrow \mathbf{t} P / X \Longrightarrow \mathbf{t} Q \\
& X \Longrightarrow \mathbf{t} P \rightarrow Q ; X \Longrightarrow \mathbf{f} Q / X \Longrightarrow \mathbf{f} P \\
& \mathbf{t} \neg I \quad X \Longrightarrow \mathbf{f} P / X \Longrightarrow \mathbf{t} \neg P \\
& \mathbf{t} \neg E \quad X \Longrightarrow \mathbf{t} \neg P / X \Longrightarrow \mathbf{f} P \\
& \mathbf{f} \wedge I \quad X \Longrightarrow \mathbf{f} P / X \Longrightarrow \mathbf{f} P \wedge Q \\
& X \Longrightarrow \mathbf{f} Q / X \Longrightarrow \mathbf{f} P \wedge Q \\
& \mathbf{f} \wedge E \quad X \Longrightarrow \mathbf{f} P \wedge Q ; X \Longrightarrow \mathbf{t} P / X \Longrightarrow \mathbf{f} Q \\
& X \Longrightarrow \mathbf{f} P \wedge Q ; X \Longrightarrow \mathbf{t} Q / X \Longrightarrow \mathbf{f} P \\
& \mathbf{f} \vee I \quad X \Longrightarrow \mathbf{f} P ; X \Longrightarrow \mathbf{f} Q / X \Longrightarrow \mathbf{f} P \vee Q \\
& \mathbf{f} \vee E \quad X \Longrightarrow \mathbf{f} P \vee Q / X \Longrightarrow \mathbf{f} P \\
& X \Longrightarrow \mathbf{f} P \vee Q / X \Longrightarrow \mathbf{f} Q \\
& \mathbf{f} \rightarrow I \quad X \Longrightarrow \mathbf{t} P ; X \Longrightarrow \mathbf{f} Q / X \Longrightarrow \mathbf{f} P \rightarrow Q \\
& \mathbf{f} \rightarrow E \quad X \Longrightarrow \mathbf{f} P \rightarrow Q / X \Longrightarrow \mathbf{t} P \\
& X \Longrightarrow \mathbf{f} P \rightarrow Q / X \Longrightarrow \mathbf{f} Q \\
& \mathbf{f} \neg I \quad X \Longrightarrow \mathbf{t} P / X \Longrightarrow \mathbf{f} \neg P \\
& \mathbf{f} \neg E \quad X \Longrightarrow \mathbf{f} \neg P / X \Longrightarrow \mathbf{t} P
\end{aligned}
$$


Table III. Introduction rules for signed sentences.

$$
\begin{array}{lll}
\frac{\mathbf{f} P}{\mathbf{t} P \rightarrow Q} \mathbf{t} \rightarrow I 1 & \frac{\mathbf{t} Q}{\mathbf{t} P \rightarrow Q} \mathbf{t} \rightarrow I 2 & \begin{array}{l}
\mathbf{t} P \\
\frac{\mathbf{f} Q}{\mathbf{f} P \rightarrow Q} \mathbf{f} \rightarrow I
\end{array} \\
\frac{\mathbf{t} P}{\mathbf{t} P \vee Q} \mathbf{t} \vee I 1 & \frac{\mathbf{t} Q}{\mathbf{t} P \vee Q} \mathbf{t} \vee I 2 & \frac{\mathbf{f} P}{\mathbf{f} Q} \\
\mathbf{t} P & \mathbf{f} \vee I \\
\frac{\mathbf{t} Q}{\mathbf{t} P \wedge Q} \mathbf{t} \wedge I & \frac{\mathbf{f} P}{\mathbf{f} P \wedge Q} \mathbf{f} \wedge I 1 & \frac{\mathbf{f} Q}{\mathbf{f} P \wedge Q} \mathbf{f} \wedge I 2 \\
\frac{\mathbf{t} P}{\mathbf{f} \neg P} \mathbf{f} \neg I & \frac{\mathbf{f} P}{\mathbf{t} \neg P} \mathbf{t} \neg I &
\end{array}
$$

Table IV. Elimination rules for signed sentences.

$$
\begin{aligned}
& \text { t } P \rightarrow Q \quad \text { t } P \rightarrow Q \\
& \frac{\mathbf{t} P}{\mathbf{t} Q} \mathbf{t} \rightarrow E 1 \frac{\mathbf{f} Q}{\mathbf{f} P} \mathbf{t} \rightarrow E 2 \\
& \frac{\mathbf{f} P \rightarrow Q}{\mathbf{t} P} \mathbf{f} \rightarrow E 1 \frac{\mathbf{f} P \rightarrow Q}{\mathbf{f} Q} \mathbf{f} \rightarrow E 2 \\
& \mathbf{t} P \vee Q \quad \text { t } P \vee Q \\
& \frac{\mathbf{f} P}{\mathbf{t} Q} \mathbf{t} \vee E 1 \quad \frac{\mathbf{f} Q}{\mathbf{t} P} \mathbf{t} \vee E 2 \\
& \frac{\mathbf{f} P \vee Q}{\mathbf{f} P} \mathbf{f} \vee E 1 \quad \frac{\mathbf{f} P \vee Q}{\mathbf{f} Q} \mathbf{f} \vee E 2 \\
& \frac{\mathbf{t} P \wedge Q}{\mathbf{t} P} \mathbf{t} \wedge E 1 \quad \frac{\mathbf{t} P \wedge Q}{\mathbf{t} Q} \mathbf{t} \wedge E 2 \\
& \text { f } P \wedge Q \quad \text { f } P \wedge Q \\
& \frac{\mathbf{t} P}{\mathbf{f} Q} \mathbf{f} \wedge E 1 \quad \frac{\mathbf{t} Q}{\mathbf{f} P} \mathbf{f} \wedge E 2 \\
& \frac{\mathbf{f} \neg P}{\mathbf{t} P} \mathbf{f} \neg E \quad \frac{\mathbf{t} \neg P}{\mathbf{f} P} \mathbf{t} \neg E
\end{aligned}
$$

and IV whose premises are exemplified by previous elements of the sequence. An intelim sequence is closed if it contains both $\mathbf{t} P$ and $\mathbf{f} P$ for some formula $P$, otherwise it is open. It can be easily shown that every closed intelim sequence can be extended to an atomically closed one, i.e. one that contains both $\mathbf{t} P$ and $\mathbf{f} P$ for some atomic formula $P$. An intelim deduction of a signed formula $\varphi$ from the set of signed formulas $X$ is an intelim sequence based on $X$ ending with $\varphi$. An intelim deduction of an unsigned formula $P$ from the set of unsigned formulas $\Gamma$ is an intelim deduction of $\mathbf{t} P$ from $\{\mathbf{t} Q \mid Q \in \Gamma\}$. We say that a signed formula $\varphi$ is intelim-deducible from a set of signed formulas $X$, 
if there is an intelim deduction of $\varphi$ from $X$. We also say that $P$ is intelim-deducible from $\Gamma$, and write $\Gamma \vdash P$, if $\mathbf{t} P$ is intelim-deducible from $\{\mathbf{t} Q \mid Q \in \Gamma\}$. Finally, an intelim refutation of a set of formulas $\Gamma$ is a closed intelim sequence $\pi$ for $\{\mathbf{t} Q \mid Q \in \Gamma\}$. When such a $\pi$ exists, we say that $\Gamma$ is intelim inconsistent and write $\Gamma \vdash$. Clearly, $\Gamma \vdash$ if and only if, for some sentence $P$, both $\Gamma \vdash P$ and $\Gamma \vdash \neg P$.

It can be easily checked that this intelim system is equivalent to the one in Table II, in the sense that it justifies exactly the same deductions. ${ }^{22}$

This system is considerably weaker than classical logic. No inference which relies on "virtual information", and is therefore non-analytic in the sense discussed in Section 6, can be justified by means of its rules.

EXAMPLE 1. $P \vee Q, P \rightarrow R, Q \rightarrow R \forall R$. Here no elimination rule can be applied to the premises. On the other hand, introduction rules can (always) be applied, but here they would lead us astray, since they would introduce formulas which are not subformulas either of the premises or of the conclusion. A rigorous argument, showing that the inference cannot be justified by the intelim rules only, requires the subformula theorem discussed in Section 8.

However, the resulting logic validates all the inferences that do not require virtual information and is still strong enough to perform a good deal of ordinary deductive reasoning. For example, the intelim sequence in Table $\mathrm{V}$ is a deduction of $V$ from the premises

$$
\{\neg U \vee S, U, S \rightarrow(R \vee \neg U), \neg(Q \wedge R), P \rightarrow Q, \neg P \rightarrow T, T \wedge R \rightarrow V\}
$$

We seem, therefore, to have found a good candidate for a solution to our Problem 2: we have a "sensible" semantics for the logical operators which (i) is weaker than classical semantics (ii) is based on informational notions, and (iii) justifies exactly those inferences that are "analytical" in the strict informational sense, that is, which do not require any appeal to virtual information. The underlying idea is that the use of virtual information in the rules which define the logical operators is responsible for the intractability of their classical meaning: this meaning cannot be fully "explicated" without an "augmentative" process that, when iterated, may force us to venture out into virtual information states which go considerably beyond the ones that are "given" in the premises.

This idea is fully confirmed by the technical results discussed in the next section. As we shall see, unlike their classical meaning, what we

\footnotetext{
${ }^{22}$ The structural rules $R$ and $W$ of Table II are built into the notions of "intelim sequence" and "intelim deduction".
}

DagostinoFloridi-kl.tex; 8/09/2008; 15:00; p.27 
Table V. An example of intelim deduction.

$\begin{array}{lll}1 & \mathbf{t} \neg U \vee S & \text { premise } \\ 2 & \mathbf{t} U & \text { premise } \\ 3 & \mathbf{t} S \rightarrow(R \vee \neg U) & \text { premise } \\ 4 & \mathbf{t} \neg Q \wedge R) & \text { premise } \\ 5 & \mathbf{t} P \rightarrow Q & \text { premise } \\ 6 & \mathbf{t} \neg P \rightarrow T & \text { premise } \\ 7 & \mathbf{t} T \wedge R \rightarrow V & \text { premise } \\ 8 & \mathbf{f} \neg U & \text { from } 2 \text { by } \mathbf{f} \neg I \\ 9 & \mathbf{t} S & \text { from } 1 \text { and } 2 \text { by } \mathbf{t} \vee E 1 \\ 10 & \mathbf{t} R \vee \neg U & \text { from } 3 \text { and } 9 \text { by } \mathbf{t} \rightarrow E 1 \\ 11 & \mathbf{t} R & \text { from } 10 \text { and } 8 \text { by } \mathbf{t} \vee E 2 \\ 12 & \mathbf{f} Q \wedge R & \text { from } 4 \text { by } \mathbf{t} \neg E \\ 13 & \mathbf{f} Q & \text { from } 12 \text { and } 11 \text { by } \mathbf{f} \wedge E 2 \\ 14 & \mathbf{f ~} P & \text { from } 5 \text { and } 13 \text { by } \mathbf{t} \rightarrow E 2 \\ 15 & \mathbf{t} \neg P & \text { from } 14 \text { by } \mathbf{t} \neg I \\ 16 & \mathbf{t} T & \text { from } 6 \text { and } 16 \text { by } \mathbf{t} \rightarrow E 1 \\ 17 & \mathbf{t} T \wedge R & \text { from } 11 \text { and } 16 \text { by } \mathbf{t} \wedge I \\ 18 & \mathbf{t ~} V & \text { from } 7 \text { and } 17 \text { by } \mathbf{t} \rightarrow E 1\end{array}$

have called "the informational meaning of the logical operators" is a tractable notion, which complies with all our requirements: (i) intelim deducibility enjoys the subformula property (all its valid inferences can be justified by means of deductions which are analytical in the syntactic sense, in accordance with our requirement (4)) and (ii) there is a feasible decision procedure to establish whether a given conclusion follows from given premises. All inferences are justified by "explication" of the informational meaning of the logical operators and such explication can be carried out in practice.

REMARK 1. It can be easily verified that the relation of intelim deducibility is a logic in Tarski's sense (see footnote. 10). However, this logic has no tautologies. This is hardly surprising, since a tautology is a sentence $P$ which is "deducible from the empty set", i.e. from the empty information state, and therefore its deduction must make essential use of virtual information, which prevents it from being analytical in the strict informational sense. ${ }^{23}$ Of course, some tautologies are easy to prove despite the argument being not strictly analytical: the distinction

\footnotetext{
${ }^{23}$ It is somehow odd that the absence of tautologies in a logical system is perceived as something in need of justification. Logicians are so acquainted with the technical notion of tautology that sometimes they fail to recognize that it is the latter, if ever, that may need justification in the light of ordinary deductive practice (which makes no use of tautologies). However, it is worth remarking that, in this respect, intelim deducibility is in good company: Belnap's four-valued logic ((Belnap Jr.,
} 
tractable/intractable does not apply to single examples but to infinite classes of examples and the relation $\emptyset \vdash P$ is (most likely) intractable. On the other hand, despite its tractability, the notion of intelim deducibility is sufficient to solve a good deal of interesting problems that may look "difficult" at first sight, but are in fact easily solved via a systematic procedure.

REMARK 2. A stronger (if still sub-classical) deducibility relation could be defined as follows: $\Gamma \vdash P$ if and only if $\Gamma \cup\{\neg P\}$ is intelim inconsistent. For instance, returning to Example 1, $R$ would become intelim deducibile from $P \vee Q, P \rightarrow R$ and $Q \rightarrow R$, since the set obtained by adding $\neg R$ to the premises is intelim inconsistent (as the reader can easily check). However, such a stronger deducibility relation would fail to satisfy the Transitivity property, since it may well be that $\Gamma \cup\{\neg P\}$ and $\Gamma \cup\{P, \neg Q\}$ are both intelim inconsistent, but $\Gamma \cup\{\neg Q\}$ is not. On the other hand, this stronger definition may still be preferred if one thinks, unlike Tarski (see footnote 10), that Transitivity is not a necessary requirement for a logical system. It is not difficult to check that even the stronger definition is weaker than classical logic. For example, the classical inference $P \vee(Q \wedge R) \vdash(P \vee Q) \wedge(P \vee R)$ cannot be justified, for the set $\{P \vee(Q \wedge R), \neg((P \vee Q) \wedge(P \vee R))$ is not intelim inconsistent. In the context of this paper we are interested in transitive deducibility relations and, therefore, shall stick to the weaker definition of $\vdash$.

\section{Subformula property and tractability}

The consequence relation of Classical Propositional Logic is closed under the ex-falso quodlibet condition:

(Ex-Falso) If $\Gamma \vdash$, then $\Gamma \vdash Q$ for every $Q$.

It is easy to verify that intelim deducibility is also closed under the (ExFalso) condition. To see this, it is sufficient to note that the following sequence of signed formulas is an intelim deduction of $Q$ from $\{P, \neg P\}$ :

$\begin{array}{lll}1 & \mathbf{t} P & \text { premise } \\ 2 & \mathbf{t} \neg P & \text { premise } \\ 3 & \mathbf{t} P \vee Q & \text { from } 1 \text { by } \mathbf{t} \vee I 1 \\ 4 & \mathbf{f} P & \text { from } 2 \text { by } \mathbf{t} \neg E \\ 5 & \mathbf{t} Q & \text { from } 3 \text { and } 4 \text { by } \mathbf{t} \vee E 1\end{array}$

1976; Belnap Jr., 1977)) is another well-known (and not unrelated) example of a logical system which does not give rise to tautologies.

DagostinoFloridi-kl.tex; 8/09/2008; 15:00; p.29 
A deduction like the one above looks "odd", in that it makes essential use of both $\mathbf{t} P$ and $\mathbf{f} P$ as if " $P$ is true" and " $P$ is false" could belong to the same information state and so, in some sense, violates the Principle of Non-Contradiction. One could reasonably claim that if we discover that our information state is inconsistent we should do something to remove the inconsistency and not trust any conclusion which can be obtained by using information which is explicitly inconsistent.

Say that a signed formula $\psi$ occurring in an intelim deduction $\pi$ of $P$ from $\Gamma$ is redundant if $\psi \neq \mathbf{t} P$ and $\psi$ is not used as a premise of any application of an intelim rule in $\pi$. Call non-redundant reduction of $\pi$ the intelim deduction of $P$ from $\Gamma$ obtained from $\pi$ by removing the redundant signed formulas. Then, we say that an intelim deduction of $P$ from $\Gamma$ is regular if its non-redundant reduction is open, and irregular otherwise. In other words, irregular deductions of $P$ from $\Gamma$ are deductions in which information which is explicitly inconsistent and recognized as such has been used to obtain a given conclusion. (Observe that whether a given intelim sequence is closed can be verified in polynomial time, and so it is feasible to recognize when a deduction is irregular.) The idea is that irregular deductions do not inform us that the conclusion "follows" from the premises, if not in a Pickwickian (classical) sense, but only that our premises are inconsistent. If we restrict ourselves to regular deductions, the ex-falso quodlibet principle no longer holds.

The following proposition states a basic normalization theorem for intelim deductions (the length $|\pi|$ of a K-intelim sequence $\pi$ is defined as the total number of symbols occurring in $\pi$ ):

PROPOSITION 1. Let $\Gamma$ be a finite set of formulas. Then:

1. every regular intelim deduction $\pi$ of $P$ from $\Gamma$ can be transformed into an intelim deduction $\pi^{\prime}$ of $P$ from $\Gamma$ such that (i) $\pi^{\prime}$ enjoys the subformula property, and (ii) $\left|\pi^{\prime}\right| \leq|\pi|$;

2. every intelim refutation $\pi$ of $\Gamma$ can be transformed into an intelim refutation $\pi^{\prime}$ of $\Gamma$ such that (i) $\pi^{\prime}$ enjoys the subformula property, and (ii) $\left|\pi^{\prime}\right| \leq|\pi|$.

The above proposition suggests that irregular intelim deductions may not be normalizable. And this is indeed the case, as shown by the intelim deduction of $Q$ from $\{P, \neg P\}$ given above, which cannot be reduced to one with the subformula property. So, in general, intelim deducibility does not enjoy the subformula property. However, one can reasonably claim that normalization fails exactly when it should, that is when we have already obtained a closed intelim sequence (which can be verified in polynomial time), and try to use two signed formulas of 
the form $\mathbf{t} P$ and $\mathbf{f} P$ (possibly together with other signed formulas in the sequence) to obtain a certain "conclusion" from them. In such a case, it seems far more sensible to take the closed intelim sequence as a refutation of the initial premises. Unlike irregular deductions, according to our Proposition 1, refutations are always normalizable. ${ }^{24}$ Observe also that Proposition 1 marks a clear distinction from normalization theorems that can be proved for full classical (or intuitionistic) logic, where normal proofs may be longer, and sometimes exponentially longer, than non-normal ones (the same holds true for cut-free proofs versus cut-based proofs in the sequent calculus).

REMARK 3. Observe that, since intelim deducibily satisfies the (exfalso) condition, $P$ is intelim deducible from $\Gamma$ if and only if either $\Gamma$ is intelim inconsistent, or there is a regular intelim deduction of $P$ from $\Gamma$. Moreover, Proposition 1 ensures that in either case we can restrict our attention to intelim sequences with the subformula property.

This remark immediately suggests a decision procedure for intelim deducibility: in order to establish whether $P$ is intelim deducible from a finite set of sentences $\Gamma$ we can (i) apply the intelim rules in all possible ways starting from $\{\mathbf{t} Q \mid Q \in \Gamma\}$ and restricting our attention to applications which preserve the subformula property; (ii) if the resulting intelim sequence is closed or contains $\mathbf{t} P$, then $P$ is intelim deducible from $\Gamma$, otherwise it is not.

We are now in a position to state our main result:

PROPOSITION 2. Intelim deducibility is a tractable problem. Whether a formula $P$ is intelim deducible from a finite set $\Gamma$ of formulas can be decided in polynomial (quadratic) time.

A feasible decision procedure is outlined in the Appendix.

\section{The informational meaning of the logical operators revisited}

In Section 4, we argued that the standard necessary and sufficient conditions that provide the equally standard truth-conditional definition of the meaning of the logical operators in classical propositional logic fail when the central notions of (classical) truth and falsity are replaced with central notions of informational nature, such as " $P$ is true' belongs

\footnotetext{
${ }^{24}$ If we add a proof rule corresponding to the ex-falso quodlibet principle, such as $\varphi ; \bar{\varphi} / \psi$ as a new structural rule, and modify the deducibility relation accordingly, then the subformula property holds in general.
}

DagostinoFloridi-kl.tex; 8/09/2008; 15:00; p.31 
to the information state $S$ " and " $P$ is false' belongs to the information state $S$ ". Just as in Section 7 we showed how the intelim approach to the semantics of the logical operators can be adjusted to find a solution to our Problem 2, in this section we make the same attempt with the truth-conditional approach.

Let us consider a standard propositional language $\mathcal{L}$, identified with the set of all its well-formed sentences, and let us recall that a valuation is a mapping of $\mathcal{L}$ into $\{0,1\}$. (Sometimes we shall speak of a valuation of a set of sentences $\Delta$, to mean a mapping of $\Delta$ into $\{0,1\}$.) If we apply to the values 0 and 1 the informational reading recalled in the previous paragraph, a valuation describes an information state, $v(P)=0$ means that the piece of information " $P$ is false" belongs to the information state in question and $v(P)=1$ means that the piece of information " $P$ is true" belongs to the information state in question. However, if they have to describe information states, rather than possible worlds, such valuations are usually partial: in a given information state some sentences may be assigned neither 1 nor 0 , reflecting our ignorance about their truth-value.

In this valuation-based approach, the intended meaning of the logical operators is usually specified by defining, within the set of all possible valuations, those which are admissible, i.e. those that comply with this intended meaning. Admissible valuations are usually defined by specifying a set of closure conditions that a valuation should satisfy. The usual conditions for the Boolean operators are the following:

C1 $v(P)=1$ if and only if $v(\neg P)=0$;

C2 $v(P \wedge Q)=1$ if and only if $v(P)=1$ and $v(Q)=1$;

C3 $v(P \vee Q)=1$ if and only if $v(P)=1$ or $v(Q)=1$;

C4 $v(P \rightarrow Q)=1$ if and only if $v(P)=0$ or $v(Q)=1$.

C5 $v(P)=0$ if and only if $v(\neg P)=1$;

C6 $v(P \wedge Q)=0$ if and only if $v(P)=0$ or $v(Q)=0$;

$\mathrm{C} 7 v(P \vee Q)=0$ if and only if $v(P)=0$ and $v(Q)=0$;

C8 $v(P \rightarrow Q)=0$ if and only if $v(P)=1$ and $v(Q)=0$.

A valuation satisfying the above conditions is said to be saturated. More specifically, we say that a valuation $v$ is upward saturated, if $v$ satisfies the above conditions in the "only-if" direction, and downward saturated if it satisfies them in the "if" direction. A Boolean valuation is a saturated valuation that satisfies the additional condition of being total, i.e. 
defined for all sentences. Observe that, for total valuations, conditions C5-C8 are redundant, in that they can be derived from conditions $\mathrm{C} 1-\mathrm{C} 4$. According to the standard view, the intended meaning of the classical logical operators is fixed by accepting only Boolean valuations as admissible. Moving from their classical to their informational meaning, not only must the requirement of total valuations be dropped, but also (as already argued in Section 4) some of the saturation properties become obviously unsound.

In particular, when represented as valuations, information states are not downward saturated. That $P \vee Q$ is assigned the value 1 by a valuation $v$ representing an information state does not imply that either $P$ is assigned 1 or $Q$ is assigned 1. Similarly, if $P \wedge Q$ is assigned 0 , this does not imply that either $P$ is assigned 0 or $Q$ is assigned 0 . In this context, therefore, admissible valuations cannot be specified by means of necessary and sufficient conditions such as C1-C8. All we can do is specify a set of constraints, which restrict the domain of all possible valuations to those which are compliant with the intended (informational) meaning of the logical operators, without requiring that these constraints have the form of necessary and sufficient conditions.

Let us call locale of a formula $P$, and denote it by $L(P)$, the set containing $P$ itself and its immediate subformulas. We now propose to define the informational meaning of a logical operator $\sharp$ by determining which valuations of $L(P)$ are not admissible for a formula $P$ containing $\sharp$ as the main operator. This is a negative way of defining this meaning. It allows us to detect valuations that are immediately forbidden to any agent who "understands" this meaning, without requiring her to engage in any complex task involving "augmentative" reasoning, i.e. the consideration of virtual information (see Section 6 above). For instance a valuation such that $v(P \vee Q)=1, v(P)=0$ and $v(Q)=0$ would clearly be inadmissible and therefore taken as part of the definition of "V".

These constraints are summarized in Table VI, where each line represents a minimal non-admissible valuation (the asterisk means that the corresponding informational value of the sentence may indifferently be true, false or undefined). A valuation $v$ is admissible if, for every formula $P, v$ does not contain any valuation of $L(P)$ that is ruled out by the accepted constraints expressing the informational meaning of the main operator of $P$. We shall denote by $\mathcal{A}$ the domain of all admissible valuations. Admissible valuations are partially ordered by the usual approximation relation $\sqsubseteq$ defined as follows: $v \sqsubseteq w$ (read " $w$ is a refinement of $v$ " or " $v$ is an approximation of $w$ ") if and only if $w$ agrees with $v$ on all the formulas for which $v$ is defined. Being a partial function, a partial valuation is a set of pairs of the form $\langle P, i\rangle$, where 
Table VI. The informational meaning of the logical operators explained by means of constraints on informational valuations. Each line represents a minimal non-admissible valuation.

\begin{tabular}{c|c}
\hline$\neg P$ & $P$ \\
\hline 1 & 1 \\
0 & 0
\end{tabular}

\begin{tabular}{c|c|c}
\hline$P \vee Q$ & $P$ & $Q$ \\
\hline 1 & 0 & 0 \\
0 & 1 & $*$ \\
0 & $*$ & 1
\end{tabular}

\begin{tabular}{c|c|c}
\hline$P \wedge Q$ & $P$ & $Q$ \\
\hline 1 & 0 & $*$ \\
1 & $*$ & 0 \\
0 & 1 & 1
\end{tabular}

\begin{tabular}{c|c|c}
\hline$P \rightarrow Q$ & $P$ & $Q$ \\
\hline 1 & 1 & 0 \\
0 & $*$ & 1 \\
0 & 0 & $*$
\end{tabular}

$P$ is a sentence in the given language and $i$ is equal to 0 or 1 . Each of these pairs can be thought of as a "piece of information" and the partial valuation itself as an attempt to put together such pieces of information in a way which is consistent with the intended meaning of the logical operators. The partial ordering $\sqsubseteq$ is a meet-semilattice ordering with a bottom element equal to $\emptyset$, the valuation which is undefined for all formulas of the language. It fails to be a lattice because the join of two admissible valuations may be inadmissible.

\section{Shallow information states}

Let us now investigate how our suggestion - that the informational meaning of the logical operators could be defined through a suitable set of constraints on valuations, rather than through the usual necessary and sufficient conditions - works in practice to characterize the set of inferences that are justified only by virtue of this meaning. In fact, Kant suggested that analytical judgements are recognized as true not only by virtue of the meaning of the relevant words (once it has been properly explicated), but also by virtue of the principle of non-contradiction. ${ }^{25}$ Here, we make the analogous suggestion that analytical inferences (in the strict sense investigated in this paper) are recognized as valid not only by virtue of the meaning of the logical words (as explicated by the appropriate constraints on admissible valuations), but also by virtue of a general consistency principle, which is as immediate to apply as the principle of non-contradiction. Let us first illustrate how this principle works in a typical example.

Consider a valuation $v$ such that $v(P \vee Q)=1$ and $v(P)=0$, while $P$ and $Q$ are both undefined. We can legitimately say that the value of $Q$

${ }^{25}$ See Section IV of the introduction to the Critique of Pure Reason 
in this valuation is implicitly determined by the values of $P \vee Q$ and $P$ and by our understanding of the meaning of $\vee$ based on the constraints specified in Table VI. For, there is no admissible refinement of $v$ such that $v(Q)=0$, since such a refinement would fail to satisfy one of the constraints that define the meaning of " $\mathrm{V}$ ". In other words, any assignment other than 1 would be immediately recognized as inconsistent by any agent that understands $V$ via the specified constraints. Notice that checking whether a possible refinement of a valuation regarding a specific formula $Q$ is admissible is a task that can be performed in linear time on the basis of local information. It involves only checking all the locales in which $Q$ is embedded. If assigning a certain value to $Q$ violates one of the constraints, which are specified in terms of the locales of formulas, then the valuation is not admissible.

Hence, if we understand the meaning of $\vee$, we are able to detect immediately that $Q$ cannot be assigned, consistently with this meaning, the value 0 , given that $P \vee Q$ has been assigned the value 1 and $P$ the value 0 . In such a situation, we can say that the information concerning the value of $Q$ is implicitly contained in our information state - in particular, in that portion of it concerning the locale of $P \vee Q$ - because the intended meaning of $\vee$ leaves us no option about this value. This is comparable, interestingly, to what happens in those easy steps of the sudoku game where the figure to be inserted in a given empty cell is dictated by the figures already inserted in the cells belonging to the regions into which the empty cell is contained. ${ }^{26}$ This is the most basic consistency principle by means of which logical inference can be justified analytically, that is, by virtue of the informational meaning of the logical operators as specified by the constraints in Table VI.

The time has come to revisit Hintikka's intuition (see Section 2). Like Hintikka, we shall also distinguish between increasing levels of informational depth, except that our distinctions apply to propositional logic. The deepest possible level coincides with Carnap and Bar-Hillel's semantic information and is a kind of potential information, which is intractable and not increased by deductive inference. By way of contrast, the most basic level, "shallow information", is tractable and is increased by deductive inference.

${ }^{26}$ A "region" in the classic version of the sudoku game is either a column, or a row or one of the four sub-squares into which the main square is divided. In our context, a "region" is simply the locale of a formula.

DagostinoFloridi-kl.tex; 8/09/2008; 15:00; p.35 
Given a valuation $v$, let us say that a piece of information ${ }^{27}\langle P, i\rangle$, with $i \in\{0,1\}$, is implicitly contained in $v$ at depth 0 if the complementary piece of information $\langle P,|1-i|\rangle$ is immediately ruled out solely by virtue of the meaning of the logical operators. If adding the piece of information $\langle P, 1\rangle(\langle P, 0\rangle)$ to a valuation $v$ makes it non-admissible, i.e. violates one of the constraints specifying the intended meaning of the main logical operator of $P$, then its complement $\langle P, 0\rangle(\langle P, 1\rangle)$ is implicitly contained in $v$ at depth 0 . A minimal requirement on an information state is that it is closed under such implicit information of depth 0 , i.e. the kind of information that immediately follows from the meaning constraints. We call information state of depth 0 or shallow information state an admissible valuation $v$ that is closed under the following condition (recall that $\mathcal{A}$ is the set of admissible valuations):

$\left(\mathrm{D}_{0}\right)$ For every formula $P \in \mathcal{L}$ :

$$
v \cup\{\langle P, i\rangle\} \notin \mathcal{A} \Longrightarrow\langle P,|1-i|\rangle \in v .
$$

In other words, if a piece of information is implicitly contained at depth 0 in a valuation $v$, then it must be explicitly contained in $v$. The proof of the following proposition is left to the reader.

PROPOSITION 3. A shallow information state is a Boolean valuation if and only if it contains a total valuation of all the atomic formulas of the language.

So, Boolean valuations can be seen as shallow information states that are closed under a Principle of Omniscience, the informational counterpart of the classical Principle of Bivalence:

For every information state $v$ and every atomic sentence $P$, either $P$ is true in $v$ or $P$ is false in $v$.

EXAMPLE 2. Consider an admissible valuation $v$ such that

1. $v(P \vee Q)=1$

2. $v(P)=0$

\footnotetext{
27 Let us say that a piece of information $\langle P, i\rangle$, with $i \in\{0,1\}$, is "correct" when the real truth value of $P$ is $i$ and "incorrect" otherwise. Strictly speaking, "information" can only be correct, "incorrect information" being in fact misinformation or disinformation. This point is not merely linguistic but conceptual, for it is crucial in order to understand the solution of the Bar-Hillel-Carnap Paradox. In this context, however, we are using "information" as synonymous with "content", which can be correct, and hence qualify as information, or incorrect, and hence qualify as mis- or disinformation.
} 
3. $v(Q \rightarrow R)=1$

4. $v(Q \rightarrow S)=1$

5. $v(\neg T \rightarrow \neg(R \wedge S))=1$

6. $v(T \wedge U)=0$.

We show that $v^{\prime}(U)=0$ for every information state of depth 0 that contains $v$, i.e. for every admissible valuation closed under $\mathrm{D}_{0}$ which contains $v$. From (i) and (ii), by the constraints on $\vee$, it follows that $v^{\prime} \cup\{\langle Q, 0\rangle\}$ would be non-admissible. So, by $\mathrm{D}_{0}$ :

\section{7. $v^{\prime}(Q)=1$.}

Then, from (vii) and (iii) it follows that $v^{\prime} \cup\{\langle R, 0\rangle\}$ would be nonadmissible by the meaming constraints on $\rightarrow$. Hence, by $\mathrm{D}_{0}$ again:

8. $v^{\prime}(R)=1$.

The remaining steps of the argument are similar and can be summarized as follows:

9. $v^{\prime}(S)=1$, by (iv), (vii), the meaning constraints on $\rightarrow$ and $\mathrm{D}_{0}$.

10. $v^{\prime}(R \wedge S)=1$, by (viii), (ix), the meaning constraints on $\wedge$ and $\mathrm{D}_{0}$.

11. $v^{\prime}(\neg(R \wedge S))=0$, by $(\mathrm{x})$, the meaning constraints on $\neg$ and $\mathrm{D}_{0}$.

12. $v^{\prime}(\neg T)=0$, by (v), (xi), the meaning constraints on $\rightarrow$ and $\mathrm{D}_{0}$.

13. $v^{\prime}(T)=1$, by (xii), the meaning constraints on $\neg$ and $\mathrm{D}_{0}$.

14. $v^{\prime}(U)=0$, by (vi), (xiii), the meaning constraints on $\wedge$ and $\mathrm{D}_{0}$.

Intuitively, a shallow or 0-depth information state represents the total information that a reasoner holds either explicitly, or implicitly on the basis of the intended meaning of the logical operators and of the basic consistency principle expressed by the closure condition $\mathrm{D}_{0}$.

Let us now say that $P$ is a 0 -depth logical consequence of $\Gamma$, and write, $\Gamma \Vdash_{0} P$ if $v(P)=1$ for every shallow information state $v$ such that $v(Q)=1$ for all $Q \in \Gamma$. We also write $\Gamma \Vdash_{0}$ when there exists no shallow information state $v$ such that $v(Q)=1$ for all $Q \in \Gamma$. In this case, we say that $\Gamma$ is inconsistent at depth 0 . The reader can easily check that all the rules of the intelim system of Tables III and IV are such that the conclusion is a 0-depth logical consequence of the premises. This implies that the following soundness theorem holds true: 
PROPOSITION 4. For every finite set $\Gamma$ of sentences and every sentence $P$, if there is an intelim deduction of $P$ from $\Gamma$, then $\Gamma \Vdash_{0}$ $P$.

The proof is left to the reader.

As for the completeness of the intelim system, there is a technical point which must be taken care of. It follows from the principle $\mathrm{D}_{0}$ above that in every shallow information state such that $v(P \vee P)=1$, it must hold true that $v(P)=1$. Similarly, in every shallow information state such that $v(P \wedge P)=0$ it must hold true that $v(P)=0$. So, the value of $P$ is by all means dictated, in both cases, by the value of $P \vee P(P \wedge P)$ and by the intended meaning of $\vee(\wedge)$ as specified by the meaning constraints. However, our intelim rules do not allow us, as they stand, to infer $\mathbf{t} P$ from $\mathbf{t} P \vee P$, or $\mathbf{f} P$ from $\mathbf{f} P \wedge P$, unless we make use of virtual information. This technical problem can be addressed in two different ways:

1. we pre-process all formulas and replace every occurrence of $P \vee P$, respectively $P \wedge P$, with $P$;

2. we introduce two new ad hoc elimination rules of the form $\mathbf{t} P \vee$ $P / \mathbf{t} P$ and $\mathbf{f} P \wedge P / \mathbf{f} P$, claiming that they descend immediately from the very meaning of $\vee$ and $\wedge$, with no need for assuming virtual information. ${ }^{28}$

Both solutions appear quite reasonable, though not terribly elegant. The first one requires restrictions on the language that do not undermine its expressive power. The second solution requires the addition of ad hoc rules that somehow spoil the harmony of the intelim approach, but are, on the other hand, perfectly justified by the aim of extracting all the information that can possibly be obtained on the sole basis of the meaning of the logical operators, without any appeal to virtual information. A more elegant solution would consist in recognizing that the traditional approach, based on standard inference rules, is perhaps not ideal to represent the flow of logical information, and revert to a less traditional approach based on logical networks, along the lines suggested in the Appendix. While this suggestion will be properly developed in a subsequent work, in the next proposition we assume that either of the two solutions outlined above has been adopted, and the notion of intelim deduction has been modified in the obvious way. Under this assumption, it can be shown that the following completeness theorem also holds true:

\footnotetext{
28 This solution is the same as the one adopted in (Finger and Gabbay, 2006) in response to a similar problem arising in their investigations into tractable subsystems of classical propositional logic.
} 
PROPOSITION 5. For every finite set $\Gamma$ of sentences and every sentence $P, \Gamma \vdash_{0} P$ implies that there is an intelim deduction of $P$ from $\Gamma$.

This completeness theorem brings us to our proposed solution to Problem 1. Let the shallow information content $\mathrm{SI}(P)$ of a sentence $P$ be defined as the lattice meet of all the shallow information states $v$ such that $v(P)=1$, that is:

$$
\mathrm{SI}(P)=\prod\{v \mid v(P)=1\}
$$

where $v$ ranges over the set of all shallow information states.

Then, we have that $P$ is intelim deducible from $\Gamma$ if and only if the shallow information content of $P$ is "contained" (in the sense of the approximation relation $\sqsubseteq$ ) into the shallow information content of (the conjunction of the sentences in) $\Gamma$. Since intelim deducibility is tractable (see Proposition 2 above), inferences that are analytical in the informational sense, according to this definition of information content, can also be recognized as valid by means of a feasible decision procedure. Moreover, such inferences are exactly those that are analytical in the semantic sense (requirement (3)), once the meaning of the logical operators is taken to be their informational meaning, discussed in Sections 7 and 9. Finally, such inferences are exactly those that are analytical in the fourth sense, "the strict informational sense" introduced in Section 6, which constitutes the central idea on which our whole approach is based.

\section{Synthetical reasoning in Classical Propositional Logic}

While the conditions concerning the meaning of the logical operators must be given once and for all, the consistency principles concerning the treatment of implicit information naturally admit of a gradual approach. We do not need to require that all implicit information is brought to light in one step, as if our computational resources were unlimited and the notion of information state insensitive to realistic computational limitations. Implicit information is, metaphorically, information that is "buried" under what one explicitly knows, and different kinds of information states may be considered, depending on how deeply rational agents - with their limited resources - are allowed to "dig" in order to bring it to light.

As far as shallow information states are concerned, we do not have far to dig in order to bring out the implicit information. This is literally on the surface, and hence any agent can pick it up. Consider, on the 
other hand, a valuation $v$ such that $v(P \vee Q)=1, v(P \rightarrow R)=1$ and $v(Q \rightarrow R)=1$, while $P, Q$ and $R$ are all undefined. In this case too one may legitimately hold that the value of $R$ (namely 1 ) is implicitly determined by the values of the other sentences. However, in order to "see" this, one has to dig a bit deeper. For one must reason along the following lines: although $P$ is not defined in $v$, any refinement $v^{\prime}$ of $v$ in which $P$ is defined must be such that either $v^{\prime}(P)=0$ or $v^{\prime}(P)=1$. In both cases, the value of $R$ is immediately determined in $v^{\prime}$ by the constraints on the meaning of the logical operators and the consistency principle $\mathrm{D}_{0}$. However, we need to consider the possible refinements $v^{\prime}$ of $v$ in which $P$ is defined, that is, we need to expand our initial information state with respect to the sentence $P$. Hence, although the value of $R$ is still implicitly determined by that of the "surrounding" sentences, bringing out this implicit information requires considering virtual information, that is, "visiting" virtual valuations that are more defined than the original one.

Our suggestion is that one can naturally define information states of different depth, depending on (i) the allowed methods for digging up implicit information and (ii) their computational cost. But then, the informational interpretation of logical consequence gives rise to different deducibility relations, depending on the depth of the information states that are used to determine whether the relation holds for a set of premises $\Gamma$ and a proposed conclusion $P$, that is depending on the depth at which the use of virtual information can be recursively allowed. In this respect, there are several options. We shall briefly describe one of the most interesting, which allows for the characterization of a hierarchy of tractable logical systems converging to classical propositional logic.

Given two (admissible) valuations $v$ and $v^{\prime}$, say that $v^{\prime}$ is a refinement of $v$ on $P$ if (i) $v \sqsubseteq v^{\prime}$ and (ii) $P$ is defined in $v^{\prime}$. Now, we call information state of depth 0 a shallow information state as defined in Section 10 . Then, for every $k \in \mathbb{N}_{+}$, we call information state of depth $k$ an admissible valuation $v$ that is closed under the following condition:

$\left(\mathrm{D}_{k}\right)$ For every formula $Q \in \mathcal{L}$, if the following condition holds true:

- there exists an atomic formula $P$ such that $v^{\prime}(Q)=i$ (with $i=0,1)$ for every information state $v^{\prime}$ of depth $k-1$ which is a refinement of $v$ on $P$,

then $v(Q)=i$.

This definition permits the use of atomic virtual information (namely, the atomic sentence $P$ ) up to a fixed depth. Then, for every $k \in \mathbb{N}$ we 
can say that $P$ is a $k$-depth logical consequence of $\Gamma$, and write $\Gamma \Vdash_{k} P$, if $v(P)=1$ for every information state $v$ of depth $k$ such that $v(Q)=1$ for all $Q \in \Gamma$. We also write $\Gamma \Vdash_{k}$ when there exists no information state $v$ of depth $k$ such that $v(Q)=1$ for all $Q \in \Gamma$. In this case, we say that $\Gamma$ is inconsistent at depth $k$. Observe that, by definition, $\Vdash_{k} \subseteq \Vdash_{k+1}$ for all $k \in \mathbb{N}$, and it is not difficult to show that the inclusion is proper, that is, $\Vdash_{k+1}$ is strictly more powerful than $\Vdash_{k}$.

The notion of intelim deducibility, defined in Section 7, can now be seen as the first level of a corresponding hierarchy, namely as intelim deducibility of depth $0\left(\vdash_{0}\right)$. For every $k \in \mathbb{N}_{+}$, an intelim sequence of depth $k$ based on a set of signed formulas $X$ is a sequence $\varphi_{1}, \ldots, \varphi_{n}$ of signed formulas such that each element $\varphi_{i}$ of the sequence either (i) is an element of $X$, or (ii) is intelim deducible at depth $k-1$ from both $\varphi_{1}, \ldots, \varphi_{i-1}, \mathbf{t} P$ and $\varphi_{1}, \ldots, \varphi_{i-1}, \mathbf{f} P$ for some atomic $P$. (The general structure of a $k$-depth intelim sequence is illustrated in Figure 1, together with an example of an intelim sequence of depth 1.) The generalization to depth $k$ of the other notions defined in Section 7 can be obtained in the obvious way. We write $\Gamma \vdash_{k} P$ when $P$ is intelim deducibile from $\Gamma$ at depth $k$ and $\Gamma \vdash_{k}$ when $\Gamma$ is intelim inconsistent at depth $k$. Note that, by definition, $\vdash_{k} \subseteq \vdash_{k+1}$. Again, $\vdash_{k+1}$ is strictly more powerful than $\vdash_{k}$.

We can show that, for every $k \in \mathbb{N}, \Gamma \vdash_{k} P$ if and only if $\Gamma \vdash_{k} P$. Moreover, each relation $\vdash_{k}$ is a logical system in Tarski's sense, that is, it satisfies the following conditions:

$$
\begin{aligned}
& \text { (Reflexivity) } \quad P \vdash_{k} P \text {, for all } P \\
& \text { (Monotonicity) If } \Gamma \vdash_{k} P \text {, then } \Gamma, Q \vdash_{k} P \text {, for all } Q \\
& \text { (Cut) If } \Gamma \vdash_{k} P \text {, and } \Gamma, P \vdash_{k} Q \text {, then } \Gamma \vdash_{k} Q
\end{aligned}
$$

Finally, every logic in this hierarchy admits of a polynomial time decision procedure:

PROPOSITION 6. Intelim deducibility of depth $k$ is a tractable problem for every fixed natural number $k$. Whether a formula $P$ is intelim deducible at depth $k$ from a finite set $\Gamma$ of formulas can be decided in polynomial time $\left(\mathcal{O}\left(n^{2 k+2}\right)\right)$.

The decision procedure can be worked out from the decision procedure for depth-0 intelim-deducibility sketched in the proof of Proposition 2 provided in the Appendix. The details of such a procedure and the proof of the upper bound will be given in a subsequent paper. As the above proposition shows, the upper bound on the complexity of the decision 

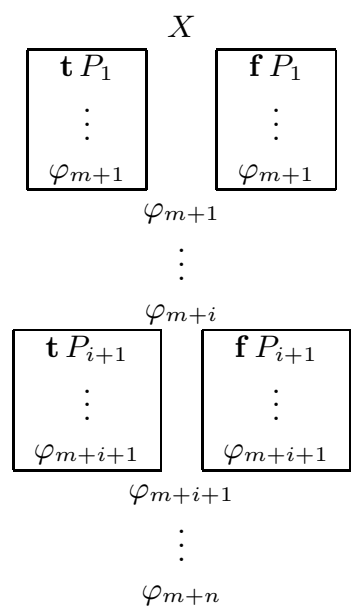

(a)

$$
\begin{aligned}
& \mathbf{t} P \vee Q \\
& \mathbf{t} P \vee(Q \rightarrow S) \\
& \mathbf{t} \neg P \vee R \\
& \mathbf{t} \neg P \vee(R \rightarrow S)
\end{aligned}
$$

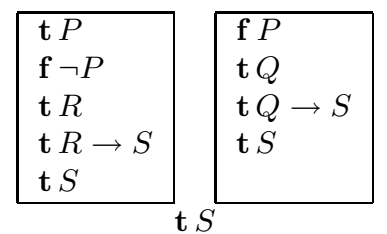

(b)

Figure 1. Figure (a) illustrates the general structure of an intelim sequence of depth $k$ based on $X=\varphi_{1}, \ldots, \varphi_{m}$. Each box leading to $\varphi_{m+j}$ (with $j=1, \ldots, n$ ) contains an intelim sequence of depth $k-1$ based on $\varphi_{1}, \ldots, \varphi_{m+j-1}$ plus the "virtual information" t $P_{j}$ or $\mathbf{f} P_{j}$, with $P_{j}$ atomic. If $\varphi_{m+j}$ is intelim deducibile at depth $k-1$ from the signed sentences which precede it, the virtual information concerning $P_{j}$ is not needed, and so there is only one intelim sequence of depth $k-1$ leading to $\varphi_{m+j}$. Figure (b) provides an example of an intelim sequence of depth 1.

procedures grows with $k$. The limit $\Vdash_{\infty}$ of this infinite sequence of logical systems, for which the upper bound is exponential, is classical propositional logic.

Although quite independently of the main problem addressed in this paper - the informativeness of classical propositional logic - the idea of approximating full classical propositional logic via a sequence of "parameterized" tractable subsystems has received considerable attention in the field of automated deduction. See, for instance, (Cadoli and Schaerf, 1992), (Dalal, 1996), (Dalal, 1998), (Crawford and Etherington, 1998). More recently Marcelo Finger (in collaboration with Renata Wasserman and Dov Gabbay) has devoted a series of papers to a systematic investigation of this problem; see, e.g., (Finger, 2004a), (Finger, 2004b), (Finger and Wasserman, 2004) and (Finger and Gabbay, 2006). The last paper addresses the problem by limiting the use of a rule corresponding to the principle of bivalence - or, equivalently, to the classical cut rule - in the context of a sequent-based presentation of the system KE ((D'Agostino and Mondadori, 1994)) whose operational rules consist of the elimination rules illustrated in Table IV. (A suggestion in this sense can be found in (D'Agostino and Mondadori, 1994, Prop. 5.6); see also (D'Agostino, 1999, Prop. 46).) This approach could 
therefore be usefully compared to the one proposed here. However, both the basis of the approximation process and the approximation method are quite different, and a detailed technical comparison would go largely beyond the scope of the present paper. ${ }^{29}$

\section{Conclusion}

Solving the scandal of deduction was never going to be easy. The reader who has kindly followed us up to this point may be reminded that we have been able to devise a strategy that we believe to be more successful than others proposed before because we have been able to rely on many previous efforts, on recent results in theoretical computer science and on equally recent insights in the philosophy of information.

By way of conclusion, it might be worth highlighting that our solutions of the Bar-Hillel-Carnap Paradox and of the Scandal of Deduction are both based on a fundamental re-adjustment of the sort of resources to which an ideal investigator may be allowed to help herself.

${ }^{29}$ We just mention a few points which mark the main differences between our approach and others that can be found in the literature:

1. Unlike (Dalal, 1996), (Dalal, 1998), (Crawford and Etherington, 1998), our approach works for full classical propositional logic, without requiring preliminary translation into any normal form.

2. Each approximation $\Vdash_{k}$ satisfies the Transitivity principle, while none of the approximations in (Dalal, 1996), (Dalal, 1998), (Crawford and Etherington, 1998), (Finger, 2004a), (Finger, 2004b), (Finger and Wasserman, 2004), (Finger and Gabbay, 2006) does. So, each $\vdash_{k}$ can be legitimately considered as a logic (see footnote 10 above) on its own, as opposed to a mere approximation.

3. The basic logic $\Vdash_{0}$ is closed under both elimination and introduction rules and so provides a full characterization of the logical operators. One can therefore claim that the classical meaning of the logical operators is defined once and for all by the basic logic, although this definition is different from the one adopted in the Gentzen tradition. The approximating logics differ from each other only in their use of atomic virtual information. By way of contrast, in (Finger and Gabbay, 2006) part of the classical meaning of the logical operators (the part which justifies the introduction rules) is missing in the basic logic and is retrieved only through the approximation process. As a consequence, their use of the classical principle of bivalence (alias the classical cut rule), which roughly speaking corresponds to our "virtual information", cannot be restricted to atomic formulas.

4. In our approach there is no fixed upper bound on the number of times in which virtual information can be introduced, or on the number of distinct formulas which can be used in this role. The fixed upper bound is only on the depth at which the recursive use of atomic virtual information is allowed. This is a general and uniform structural principle concerning our computational limitations and does not depend on some more or less arbitrary restriction on the formulas to be used as virtual information. 
In the case of the Paradox, more is available than was previously expected. This "more" comes in the form of a strongly semantic conception of information as encapsulating truthfulness. If our investigator cannot use this strong notion of information, she is inevitably caught in the paradox.

In the case of the Scandal, we have argued that the problem was caused by the very natural and understandable oversight that too much was being allowed. This "too much" comes in the form of "virtual information". Allow the investigator to rely on it, and she will never be able to explain why and in what way logic might be informative after all.

In short, our twofold proposal may be summarised by saying that a more balanced, informational diet (more truth, less virtual information) can resolve both the Paradox and the Scandal. The headache is in the details.

\section{Appendix}

Proof of Proposition 1. An occurrence of a signed formula $\varphi$ is a detour in a $K$-intelim deduction $\pi$ if it is at the same time the conclusion of an application of an introduction rule and the major premise of an application of an elimination rule. Then, we can show that if $\pi$ is a regular $K$-intelim deduction of $P$ from $\Gamma, \pi$ can be shortened to a $K$-intelim deduction of $P$ from $\Gamma$ that contains no detours.

We can assume without loss of generality that $\pi$ is non-redundant. Suppose $\pi$ contains a detour, say an occurrence of $T P \vee Q$. Then this must be at the same time the conclusion of an introduction and the major premise of an elimination. Suppose the premise of the introduction is $\mathbf{t} P(\mathbf{t} Q)$; if the conclusion of the elimination is also $\mathbf{t} P$ $(\mathbf{t} Q)$, than the latter is clearly redundant and can be removed from the deduction; if the conclusion of the elimination is $\mathbf{t} Q(\mathbf{t} P), \pi$ must contain also $\mathbf{f} P(\mathbf{f} Q)$. So $\pi$ is closed and therefore is not a regular deduction of $P$ from $\Gamma$. So, the detour can always be eliminated and the resulting sequence is always shorter. Now, say that a signed formula $\varphi=\mathbf{s} Q$, with $\mathbf{s} \in\{\mathbf{t}, \mathbf{f}\}$, is spurious if $Q$ is not a subformula of some formula in $\Gamma$ or of $P$. Suppose $\psi$ is a spurious formula of maximum complexity occurring in a $K$-intelim deduction $\pi$ of $P$ from $\Gamma$. Then, $\psi$ can occur in $\pi$ only as a result of a detour. Since detours can be eliminated from deductions, we eventually obtain a deduction without spurious formulas. The formal proof is by induction on $\langle k, n\rangle$, where $k$ is the maximum complexity of the spurious formulas occurring in $\pi$ and $n$ is the number of spurious formulas of complexity $k$. So, regular $K$ -

DagostinoFloridi-kl.tex; 8/09/2008; 15:00; p.44 
intelim deductions enjoy the subformula property. A similar argument shows that $K$-intelim refutations enjoy the subformula property.

Proof of Proposition 2. Proposition 1 implies that, when deciding whether $\Gamma \Vdash_{0} P$ we can restrict our attention to intelim deductions satisfying the subformula property without loss of deductive power. We now sketch a tractable decision procedure for intelim-deducibility which exploits the subformula property.

Given a finite set of formulas $\Gamma$, let $\Gamma^{*}$ be the set of all subformulas of formulas in $\Gamma$. It is easy to check that the size of this set is linear in the size of (the total number of symbols occurring in) $\Gamma$. Let $G(\Gamma)$ be the subformula graph for $\Gamma$, that is the oriented graph $\langle V, E\rangle$ such that $V=\Gamma^{*}$ and $\langle P, R\rangle \in E$ if and only if $P$ is an immediate subformula of $R$. Given a node $P$ in $G(\Gamma)$, we call neighbours of $P$ all the nodes $R$ such that $\langle P, R\rangle \in E$ (upper neighbours) or $\langle R, P\rangle \in E$ (lower neighbours).

A partial information graph for $\Gamma$ is a pair $\mathcal{G}=\langle G(\Gamma), \lambda\rangle$, where $\lambda$ is a partial function mapping nodes of $G(\Gamma)$ into $\{0,1\}$. We shall say that a node $P$ is labelled when $\lambda(P)$ is defined, and unlabelled otherwise. Intuitively, $\lambda(P)=1$ means "we hold the information that $P$ is true" and $\lambda(P)=0$ means "we hold the information that $P$ is false". So, the interpretation of a node $P$ labelled with 1 is the same as that of the signed formula $\mathbf{t} P$. Similarly, the interpretation of a node $P$ labelled with 0 is the same as that of the signed formula $\mathbf{f} P$. Therefore, we shall say that labelled nodes represent the associated signed formulas. On the other hand, when $\lambda(P)$ is undefined, this means that we do not hold any information about the truth-value of $P$.

In what follows we describe a procedure to update the labelling function $\lambda$ so as to extract all the implicit information of depth 0 contained in the graph. This procedure, that we call "UPDATE" is based on two sub-procedures, "INTELIM-UP" and "INTELIM-DOWN", that are called by UPDATE as modules.

\section{Procedure: INTELIM-UP $(P)$}

When applied to a formula $P$ for which the labelling function $\lambda$ is defined, the procedure visits each upper neighbour $R$ of $P$ exactly once and either locally updates $\lambda$ in accordance with the intelim rules or halts when the required updating is ruled out by the conditions on admissible valuations.

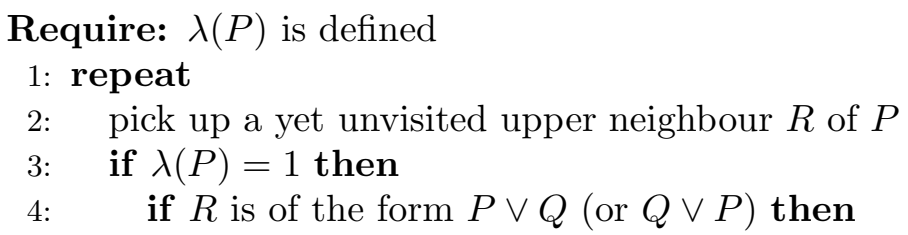


if $\lambda(R)$ is undefined then update $\lambda$ by setting $\lambda(R)=1$

else if $\lambda(R)=0$ then

STOP: $\lambda$ is not admissible

else

do nothing

end if

end if

if $R$ is of the form $P \wedge Q$ (or $Q \wedge P)$ then

if $\lambda(R)$ is undefined and $\lambda(Q)=1$ then update $\lambda$ by setting $\lambda(R)=1$

else if $\lambda(R)=0$ and $\lambda(Q)$ is undefined then update $\lambda$ by setting $\lambda(Q)=0$

else if $\lambda(R)=0$ and $\lambda(Q)=1$ then STOP: $\lambda$ is not admissible

else

do nothing

end if

end if

if $R$ is of the form $P \rightarrow Q$ then

if $\lambda(R)$ is undefined and $\lambda(Q)=0$ then update $\lambda$ by setting $\lambda(R)=0$

else if $\lambda(R)=1$ and $\lambda(Q)$ is undefined then update $\lambda$ by setting $\lambda(Q)=1$

else if $\lambda(R)=1$ and $\lambda(Q)=0$ then STOP: $\lambda$ is not admissible

else

do nothing

end if

end if

if $R$ is of the form $Q \rightarrow P$ then

if $\lambda(R)$ is undefined then update $\lambda$ by setting $\lambda(R)=1$

else if $\lambda(R)=0$ then

STOP: $\lambda$ is not admissible

else

do nothing

end if

end if

if $R$ is of the form $\neg P$ then

if $\lambda(R)$ is undefined then update $\lambda$ by setting $\lambda(R)=0$

else if $\lambda(R)=1$ then 
48:

STOP: $\lambda$ is not admissible else

do nothing end if

end if

else if $\lambda(P)=0$ then

if $R$ is of the form $P \vee Q$ (or $Q \vee P$ ) then

if $\lambda(R)$ is undefined and $\lambda(Q)=0$ then update $\lambda$ by setting $\lambda(R)=0$

else if $\lambda(R)=1$ and $\lambda(Q)$ is undefined then update $\lambda$ by setting $\lambda(Q)=1$

else if $\lambda(R)=1$ and $\lambda(Q)=0$ then STOP: $\lambda$ is not admissible else

do nothing end if end if

if $R$ is of the form $P \wedge Q$ (or $Q \wedge P$ ) then

if $\lambda(R)$ is undefined then update $\lambda$ by setting $\lambda(R)=0$

else if $\lambda(R)=1$ then

STOP: $\lambda$ is not admissible

else

do nothing

end if

end if

if $R$ is of the form $P \rightarrow Q$ then

if $\lambda(R)$ is undefined then update $\lambda$ by setting $\lambda(R)=1$

else if $\lambda(R)=0$ then

STOP: $\lambda$ is not admissible

else

do nothing

end if

end if

if $R$ is of the form $Q \rightarrow P$ then

if $\lambda(R)$ is undefined and $\lambda(Q)=1$ then update $\lambda$ by setting $\lambda(R)=0$

else if $\lambda(R)=1$ and $\lambda(Q)$ is undefined then update $\lambda$ by setting $\lambda(Q)=0$

else if $\lambda(R)=1$ and $\lambda(Q)=1$ then

STOP: $\lambda$ is not admissible

else 


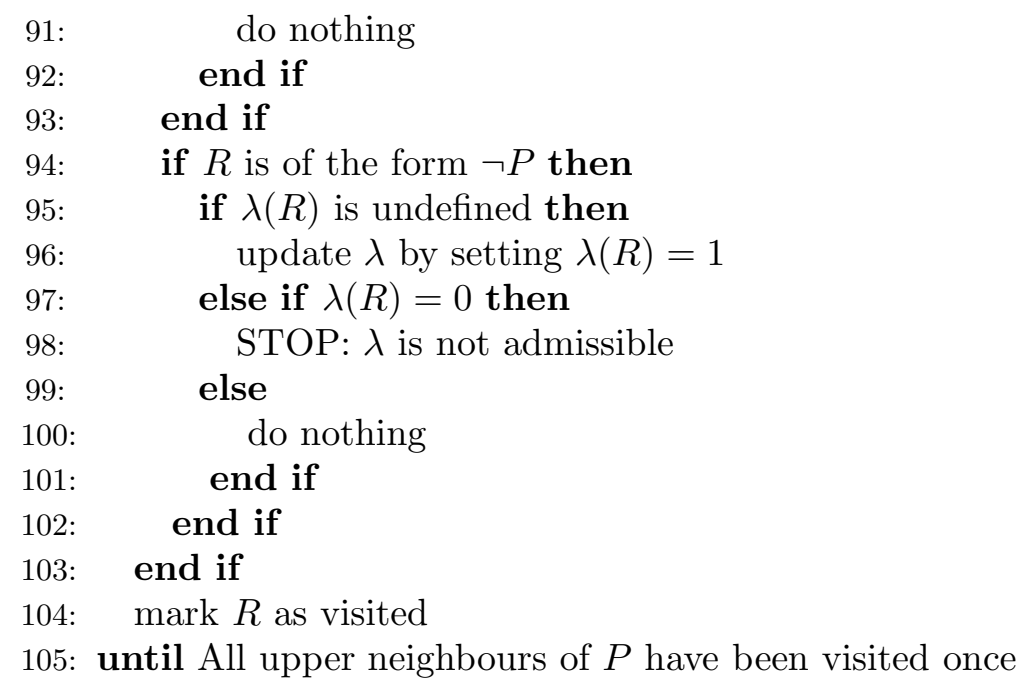

\section{Procedure: INTELIM-DOWN $(P)$}

When applied to a formula $P$ for which the labelling function $\lambda$ is defined, the procedure visits each lower neighbour $R$ of $P$ exactly once and either updates $\lambda$ in accordance with the intelim rules or halts when the required updating is ruled out by the conditions on admissible valuations.

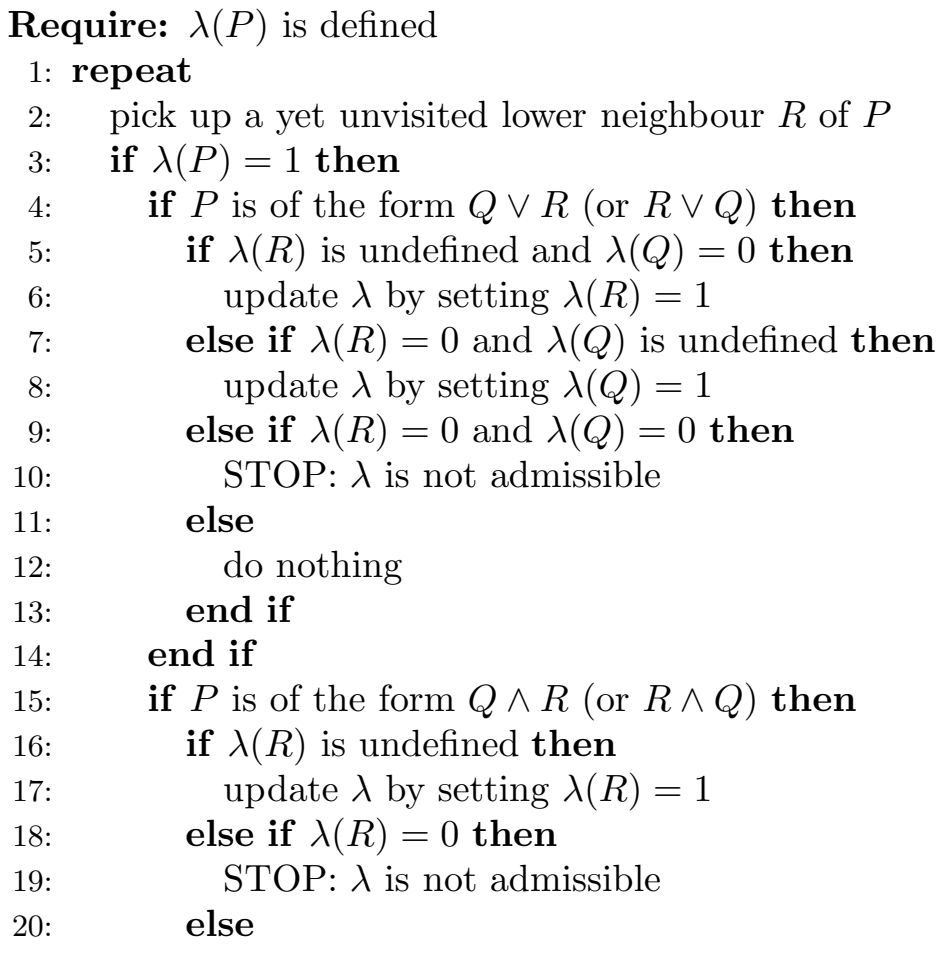


21:

22 :

23 .

59:

60:

61:

62 :

63 : do nothing

end if

end if

if $P$ is of the form $Q \rightarrow R$ then

if $\lambda(R)$ is undefined and $\lambda(Q)=1$ then update $\lambda$ by setting $\lambda(R)=1$

else if $\lambda(R)=0$ and $\lambda(Q)$ is undefined then update $\lambda$ by setting $\lambda(Q)=0$

else if $\lambda(R)=0$ and $\lambda(Q)=1$ then

STOP: $\lambda$ is not admissible

else

do nothing

end if

end if

if $P$ is of the form $R \rightarrow Q$ then

if $\lambda(R)=1$ and $\lambda(Q)$ is undefined then update $\lambda$ by setting $\lambda(Q)=1$

else if $\lambda(R)$ is undefined and $\lambda(Q)=0$ then update $\lambda$ by setting $\lambda(R)=0$

else if $\lambda(R)=1$ and $\lambda(Q)=0$ then

STOP: $\lambda$ is not admissible

else

do nothing

end if

end if

if $P$ is of the form $\neg R$ then

if $\lambda(R)$ is undefined then update $\lambda$ by setting $\lambda(R)=0$

else if $\lambda(R)=1$ then

STOP: $\lambda$ is not admissible

else

do nothing

end if

end if

else if $\lambda(P)=0$ then

if $P$ is of the form $Q \vee R$ (or $R \vee Q$ ) then

if $\lambda(R)$ is undefined then

update $\lambda$ by setting $\lambda(R)=0$

else if $\lambda(R)=1$ then

STOP: $\lambda$ is not admissible

else

do nothing

end if 


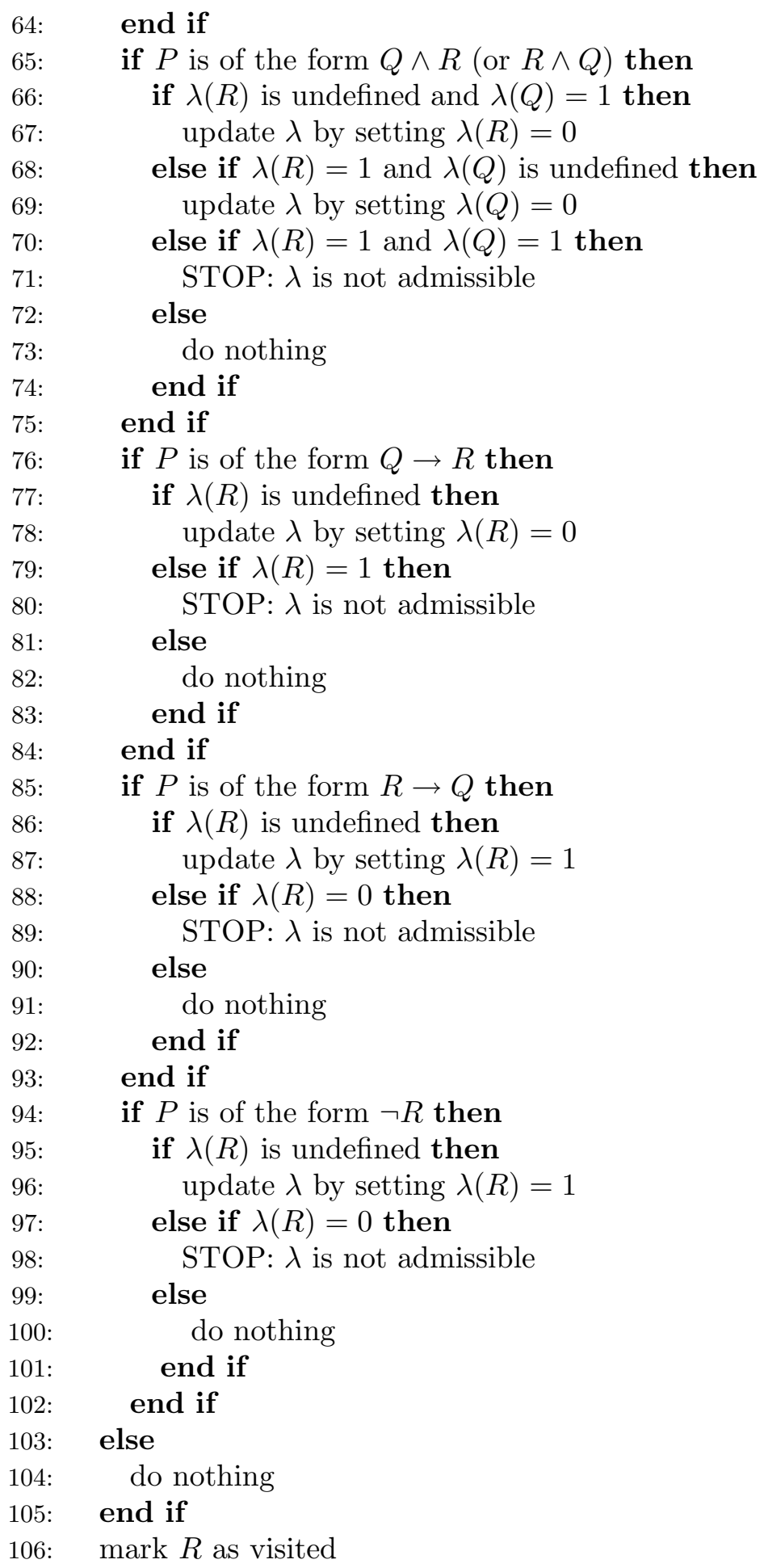


107: until All lower neighbours of $P$ have been visited once

The sub-procedures INTELIM-UP and INTELIM-DOWN are called by the main procedure UPDATE described below.

Say that a partial information graph for $\Gamma$ is saturated if its labelling function $\lambda$ represents a valuation of $\Gamma^{*}$ which is closed under condition $D_{0}$ of section 10 , that is, a shallow information state restricted to the formulas in $\Gamma^{*}$. Observe that this is equivalent to saying that the $\lambda$ function is closed under the intelim rules, once the application of an intelim rule is defined in the obvious way: if a partial information graph contains labelled nodes that represent the premises of a rule application whose conclusion is a signed formula $\mathbf{s} P$ (with $\mathbf{s}=\mathbf{t}$ or $\mathbf{f}$ ) such that $P$ is in $\Gamma^{*}$, then $\lambda(P)=1$ if $\mathbf{s}=\mathbf{t}$ and $\lambda(P)=0$ if $\mathbf{s}=\mathbf{f}$.

\section{Procedure: UPDATE $(\mathcal{G})$}

When applied to a partial information graph $\mathcal{G}$, this procedure visits each labelled node exactly once and and runs the INTELIM-UP and INTELIM-DOWN sub-procedures on it. It outputs the (unique) saturated refinement of the initial graph or halts if no saturated refinement exists.

repeat

pick up a yet unvisited labelled node $P$

apply both INTELIM-UP and INTELIM-DOWN to $P$

if both procedures terminate with an admissible $\lambda$ then

mark $P$ as visited

\section{else}

STOP: the initial graph represents an inconsistent set of signed formulas

\section{end if}

until all labelled nodes have been visited once

Figure 2 shows the initial intelim graph for the set of signed formulas:

$$
\{\mathbf{t} P \vee Q, \mathbf{t} P \rightarrow R \wedge S, \mathbf{t} Q \rightarrow \neg(T \wedge \neg S), \mathbf{t} \neg S\},
$$

while the final graph returned by the UPDATE procedure is shown in Figure 3 The steps of the UPDATE procedure can be immediately read as applications of intelim rules and, therefore, as producing an intelim sequence based on the set of signed formulas $\{\mathbf{t} Q \mid Q \in \Gamma\}$. It is simple to show that the final output graph is saturated (or, equivalently, closed under the intelim rules) and, therefore, the sequence associated with it contains all possible applications of intelim rules, starting from the initial set of signed formulas, whose conclusions have the form s $Q$ with $Q$ a subformula of $P$ or of some formula in $\Gamma$. Given the subformula 


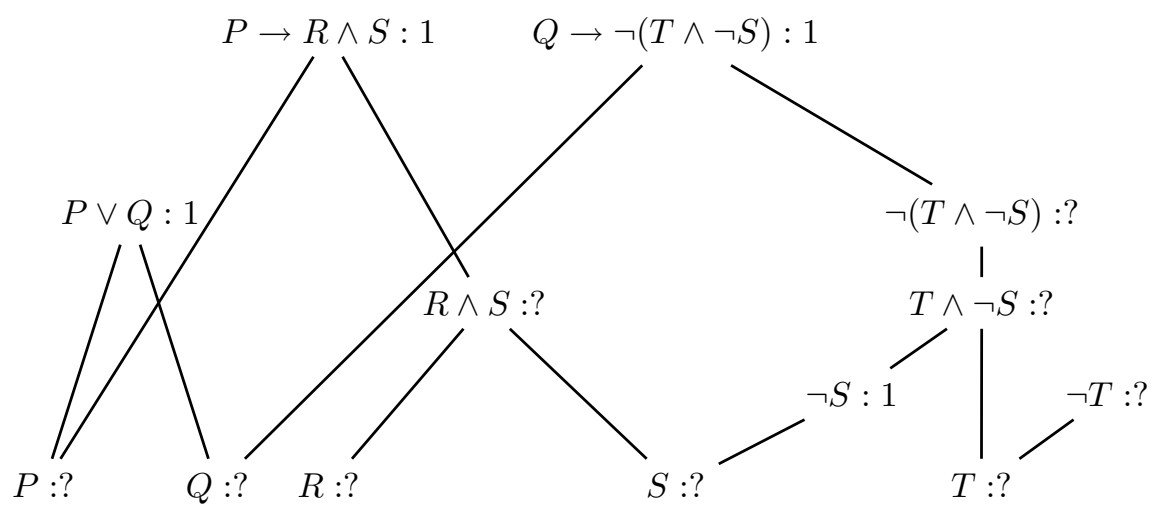

Figure 2. Initial intelim graph.

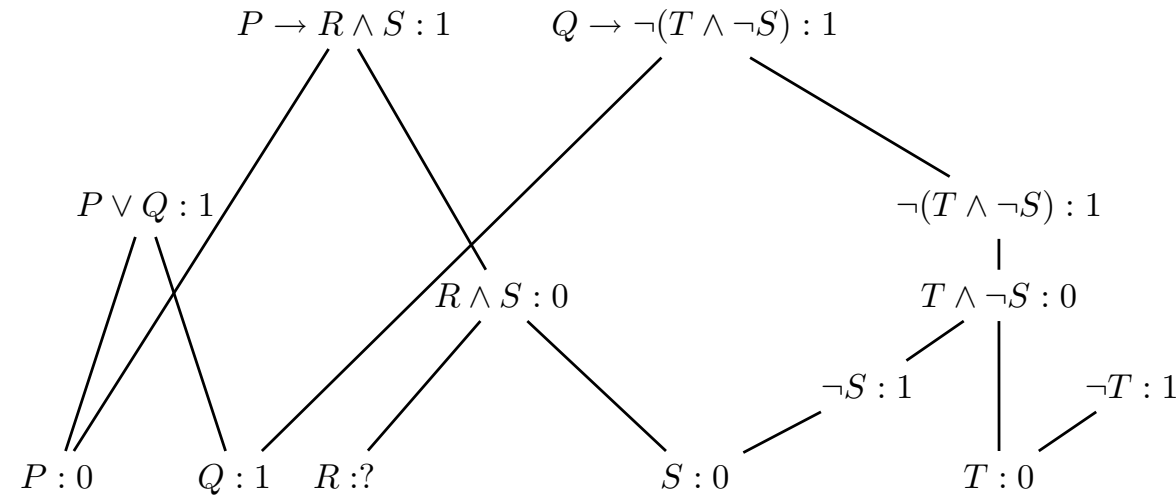

Figure 3. Output of the UPDATE procedure run on the initial graph represented in Figure 2.

property of intelim deductions (Proposition 1) this is all we need. We sketch the proof in one case and leave the rest to the reader.

Suppose that the final output graph contains nodes $P$ and $P \vee Q$ such that $\lambda(P)=0$ and $\lambda(P \vee Q)=1$; then it must be the case that $\lambda(Q)=1$. To establish this fact one can reason as follows. Since both $P$ and $P \vee Q$ are labelled, they must have been visited by the UPDATE procedure. Suppose that $P$ was visited before $P \vee Q$ and that $\lambda$ was yet undefined for $P \vee Q$ at the time $P$ was visited, so that the relevant instruction of the INTELIM-UP procedure (lines 57-58) could not be applied and $\lambda$ was not updated on $Q$. However, at the time in which $P \vee Q$ is visited by the UPDATE procedure, the conditions of the relevant instruction of INTELIM-DOWN (lines 5-6 modulo the obvious substitutions of variables) are met, and therefore $\lambda$ can be updated by setting $\lambda(Q)=1$. On the other hand, suppose that $P \vee Q$ was visited before $P$ and $\lambda(P)$ was yet undefined at that time, so that the relevant instruction of the INTELIM-DOWN procedure (lines 5-6 
modulo the obvious substitutions of variables) could not be applied and $\lambda$ was not updated on $Q$. As before, at the time in which $P$ is visited, the conditions for the relevant instruction of the INTELIM-UP procedure (lines 57-58) are met and, therefore, $\lambda$ can be updated by setting $\lambda(Q)=1$. All the remaining cases are similar.

Now, in order to establish whether $\Gamma \Vdash_{0} P$ it is sufficient to perform the following steps:

1: construct a partial information graph for $\Gamma \cup\{P\}$, that is a graph $\mathcal{G}=\langle G(\Gamma \cup\{P\}), \lambda\rangle$, such that $\lambda(Q)=1$ for all $Q \in \Gamma$ and left undefined everywhere else;

2: run the UPDATE procedure on $\mathcal{G}$;

3: if the resulting $\lambda$ is not admissible or $\lambda(P)=1$ in the output graph then

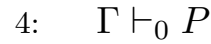

5: else

6: $\quad \Gamma \nvdash_{0} P$.

7: end if

As for the complexity of this decision procedure, observe that the size of the subformula graph for $\Gamma \cup\{P\}$ is $\mathcal{O}(n)$ where $n$ is the size $(=$ the number of symbols) of $\Gamma \cup\{P\}$. Hence, the first step requires time $\mathcal{O}\left(n^{2}\right)$. The second step involves, for every node $P$ of $\mathcal{G}$, visiting all the neighbours of $P$ exactly once and applying the relevant intelim rule where applicable; since there are at most $n$ neighbours for each node, and a fixed number of basic instructions to execute to apply the relevant intelim rules, the whole procedure runs in $\mathcal{O}\left(n^{2}\right)$ steps.

Proof of Proposition 5. Given a finite set of sentences $\Gamma$ we construct an infinite intelim sequence based on $\{\mathbf{t} Q \mid Q \in \Gamma\}$ as follows. Suppose $\Gamma=\left\{Q_{1}, \ldots, Q_{m}\right\}$ and consider an arbitrary enumeration of all the sentences of the language $\mathcal{L}$. Now, let $\pi_{0}=\mathbf{t}\left(Q_{1}\right), \ldots, \mathbf{t}\left(Q_{m}\right)$, and let $\pi_{k+1}=\pi_{k}, \mathbf{s} R$, where $R$ is the first sentence the enumeration of $\mathcal{L}$ such that (i) $\mathbf{s} R$ is the conclusion of an application of an intelim rule to signed sentences in $\pi_{k}$ and (ii) $\mathbf{s} R$ is not already in $\pi_{k}$. Finally, let $\pi=\bigcup_{i=0}^{\infty} \pi_{i}$. Observe that, if there is no intelim deduction of $P$ from $\Gamma$, then (i) $\pi$ must be open (since if $\pi$ is closed, then it contains a finite subsequence which is still a closed intelim sequence based on the same initial set of signed sentences) and (ii) $\mathbf{t} P$ cannot be in $\pi$ (otherwise, $\pi$ would contain a finite subsequence which is an intelim deduction of $P$ from $\Gamma$ ). Consider then the partial function $v$ from $\mathcal{L}$ into $\{0,1\}$ defined as follows:

1. $v(Q)=1$ if and only if $\mathbf{t} Q$ is in $\pi$;

2. $v(Q)=0$ if and only if $\mathbf{f} Q$ is in $\pi$;

DagostinoFloridi-kl.tex; 8/09/2008; 15:00; p.53 
3. $v(Q)$ is undefined otherwise.

It is not difficult to check that $v$ is a shallow information state that verifies all the sentences in $\Gamma$ and does not verify $Q$.

\section{Acknowledgements}

We wish to thank Marcelo Finger, Dov Gabbay, Hykel Hosni, Massimo Mugnai, Sebastian Sequoiah-Grayson and two anonymous referees for several helpful comments.

\section{References}

Bar-Hillel, Y. (ed.): 1964, Language and Information: Selected Essays on Their Theory and Application. Reading, Mass. and London: Addison-Wesley.

Belnap Jr., N. D.: 1976, 'How a computer should think'. In: G. Ryle (ed.): Contemporary Aspects of Philosophy. Oriel Press, pp. 30-55.

Belnap Jr., N. D.: 1977, 'A useful four-valued logic'. In: J. M. Dunn and G. Epstein (eds.): Modern uses of multiple-valued logics. Dordrecht: Reidel, pp. 8-37.

Bendall, K.: 1978, 'Natural Deduction, Separation, and the Meaning of the Logical Operators'. Journal of Philosophical Logic 7, 245-276.

Bremer, M. and D. Cohnitz: 2004, Information and Information Flow. An Introduction. Frankfurt and Lancaster: Ontos Verlag.

Cadoli, M. and M. Schaerf: 1992, 'Tractable Reasoning via Approximation'. In: Proceedings of the AAAI Workshop on Tractable Reasoning. pp. 12-15.

Carnap, R. and Y. Bar-Hillel: 1953, 'An Outline of a Theory of Semantic Information'. In (Bar-Hillel, 1964), pp. 221-74.

Cellucci, C.: 1988, 'Efficient Natural Deduction'. In: C.Cellucci and G. Sambin (eds.): Temi e prospettive della logica e della filosofia della scienza contemporanee, Vol. I. Bologna, pp. 29-57, CLUEB.

Cellucci, C.: 1995, 'On Quine's Approach to Natural Deduction'. In: P. Leonardi and M. Santambrogio (eds.): On Quine: New Essays. Cambridge University Press, pp. 314-335.

Cook, S. A.: 1971, 'The complexity of theorem-proving procedures'. In: STOC '71: Proceedings of the third annual ACM symposium on Theory of computing. New York, NY, USA, pp. 151-158, ACM Press.

Corcoran, J.: 1998, 'Information-Theoretic Logic'. In: L. V.-F. C. Martnez, U. Rivas (ed.): Truth in Perspective. Aldershot: Ashgate Publishing Limited, pp. 113-135.

Crawford, J. M. and D. W. Etherington: 1998, 'A Non-Deterministic Semantics for Tractable Inference'. In: AAAI/IAAI. pp. 286-291.

D'Agostino, M.: 1999, 'Tableaux Methods for Classical Propositional Logic'. In: M. D'Agostino, D. Gabbay, R. Hähnle, and J. Posegga (eds.): Handbook of Tableaux Methods. Kluwer Academic Publishers, pp. 45-123.

DagostinoFloridi-kl.tex; 8/09/2008; 15:00; p.54 
D’Agostino, M.: 2005, 'Classical Natural Deduction.'. In: S. N. Artëmov, H. Barringer, A. S. d'Avila Garcez, L. C. Lamb, and J. Woods (eds.): We Will Show Them! (1). College Publications, pp. 429-468.

D'Agostino, M. and M. Mondadori: 1994, 'The taming of the cut'. Journal of Logic and Computation 4, 285-319.

D'Agostino, M. and M. Mondadori: 1999, 'La logica è davvero analitica?'. Bollettino Filosofico, Università della Calabria $\mathbf{1 5}$.

Dalal, M.: 1996, 'Anytime families of tractable propositional reasoners'. In: Proceedings of the Fourth International Symposium on AI and Mathematics (AI/MATH-96), 42-45.

Dalal, M.: 1998, 'Anytime Families of Tractable Propositional Reasoners'. Annals of Mathematics and Artificial Intelligence 22, 297-318.

Dretske, F. I.: 1981, Knowledge and the Flow of Information. Oxford: Blackwell. Reprinted in 1999 (Stanford, CA: CSLI Publications).

Dummett, M.: 1978, Truth and other Enigmas. London: Duckworth.

Dummett, M.: 1991, The logical basis of metaphysics. London: Duckworth.

Finger, M.: 2004a, 'Polynomial Approximations of Full Propositional Logic via Limited Bivalence.'. In: 9th European Conference on Logics in Artificial Intelligence (JELIA 2004), Vol. 3229 of Lecture Notes in Artificial Intelligence. pp. 526-538, Springer.

Finger, M.: 2004b, 'Towards polynomial approximations of full proposi- tional logic'. In: A. L. Bazzan and S. Labidi (eds.): XVII Brazilian Symposium on Artificial Intel ligence (SBIA 2004), Vol. 3171 of Lecture Notes in Artificial Intel lingence. pp. 11-20, Springer.

Finger, M. and D. Gabbay: 2006, 'Cut and Pay'. Journal of Logic, Language and Information 15(3), 195-218.

Finger, M. and R. Wasserman: 2004, 'Approximate and limited reasoning: Semantics, proof theory, expressivity and control'. Journal of Logic and Computation 14(2), 179-204.

Floridi, L.: 2004, 'Outline of a Theory of Strongly Semantic Information'. Minds and Machines 14(2), 197-222.

Floridi, L.: 2005, 'Is Information Meaningful Data?'. Philosophy and Phenomenological Research 70(2), 351-370.

Gentzen, G.: 1935, 'Unstersuchungen über das logische Schliessen'. Math. Zeitschrift 39, 176-210. English translation in (Szabo, 1969).

Hempel, C.: 1945, 'Geometry and Empirical Science'. American Mathematical Monthly $\mathbf{5 2 .}$

Hintikka, J.: 1973, Logic, language games and information. Kantian themes in the philosophy of logic. Oxford: Clarendon Press.

Leblanc, H.: 1966, 'Two Shortcomings of Natural Deduction'. Journal of Philosophy LXIII, 29-37.

Popper, K.: 1934, Logik Der Forschung : Zur Erkenntnistheorie Der Modernen Naturwissenschaft. Wien: J. Springer. Eng. tr. The Logic of Scientific Discovery (London: Hutchinson, 1959).

Sequoiah-Grayson, S.: 2008, 'The Scandal of Deduction. Hintikka on the Information Yield of Deductive Inferences'. The Journal of Philosophical Logic 37(1), 67-94.

Shannon, C. and W. Weaver: 1949, The Mathematical Theory of Communication. Urbana: University of Illinois Press. Foreword by R.E. Blahut and B. Hajek.

Smullyan, R. M.: 1965, 'Analytic Natural Deduction'. Journal of Symbolic Logic 30, $123-139$. 
Szabo, M. (ed.): 1969, The Collected Papers of Gerhard Gentzen. Amsterdam: North-Holland.

Tennant, N.: 1990, Natural Logic. Edinburgh University Press.

Weir, A.: 1986, 'Classical Harmony'. Notre Dame Journal of Formal Logic 27(4), 459-482. 